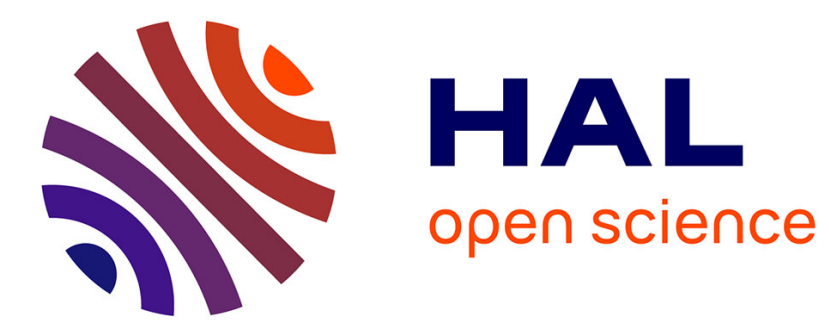

\title{
Optic Flow Based Autopilots: Speed Control and Obstacle Avoidance
}

Nicolas Franceschini, Franck Ruffier, Julien Serres

\section{To cite this version:}

Nicolas Franceschini, Franck Ruffier, Julien Serres. Optic Flow Based Autopilots: Speed Control and Obstacle Avoidance. Flying Insects and Robots, Springer Berlin Heidelberg, pp.29-50, 2009, 10.1007/978-3-540-89393-6_3 . hal-02294508

\section{HAL Id: hal-02294508 \\ https://hal-amu.archives-ouvertes.fr/hal-02294508}

Submitted on 23 Sep 2019

HAL is a multi-disciplinary open access archive for the deposit and dissemination of scientific research documents, whether they are published or not. The documents may come from teaching and research institutions in France or abroad, or from public or private research centers.
L'archive ouverte pluridisciplinaire HAL, est destinée au dépôt et à la diffusion de documents scientifiques de niveau recherche, publiés ou non, émanant des établissements d'enseignement et de recherche français ou étrangers, des laboratoires publics ou privés. 


\title{
Optic flow based autopilots: speed control and obstacle avoidance
}

Nicolas Franceschini, Franck Ruffier and Julien Serres

\begin{abstract}
:
The explicit control schemes presented here explain how insects may navigate on the sole basis of optic flow (OF) cues without requiring any distance or speed measurements: how they take off and land, follow the terrain, avoid the lateral walls in a corridor and control their forward speed automatically. The optic flow regulator, a feedback system controlling either the lift, the forward thrust or the lateral thrust, is described. Three OF regulators account for various insect flight patterns observed over the ground and over still water, under calm and windy conditions and in straight and tapered corridors. These control schemes were simulated experimentally and/or implemented onboard two types of aerial robots, a micro helicopter $(\mathrm{MH})$ and a hovercraft $(\mathrm{HO})$, which behaved much like insects when placed in similar environments. These robots were equipped with opto-electronic $O F$ sensors inspired by our electrophysiological findings on houseflies' motion sensitive visual neurons. The simple, parsimonious control schemes described here require no conventional avionic devices such as range finders, groundspeed sensors or GPS receivers. They are consistent with the the neural repertoire of flying insects and meet the low avionic payload requirements of autonomous micro aerial and space vehicles.
\end{abstract}

Nicolas Franceschini, Franck Ruffier and Julien Serres

Biorobotics Lab, Institute of Movement Science, CNRS \& Univ of the Mediterranean, Marseille, France nicolas.franceschini@univmed.fr, franck.ruffier@univmed.fr, julien.serres@univmed.fr 


\section{Introduction}

When an insect is flying forwards, an image of the ground texture and any lateral obstacles scrolls backwards across the ommatidia of its compound eye. This flowing image set up by the animal's own forward motion is called the Optic Flow (OF). Recent studies have shown that freely flying insects use the OF to avoid collisions [98,11,92], follow a corridor [55,87,3,4,83], cruise and land $[97,88,81,90]$. The OF can be described as a vector field where each vector gives the direction and magnitude of the angular velocity at which any point in the environment moves relative to the eye [56]. Several authors have attempted to model the control systems at work in insects during free flight, focusing on specific aspects such as speed control [16, 88], distance or speed servoing $[58,18,22,45]$, course control and saccadic flight behavior $[41,69,21]$. Freely flying flies navigate by making pure translations alternating with fast, saccade-like turns $[41,98,11,96]$. This idiosyncratic flight behavior was interpreted $[98,11]$ as an active means of reducing the image flow to its translational component, which specifies the relative distances to objects [56].

A biorobotic project was launched in the mid 1980's to investigate how a fly could possibly navigate and avoid collisions based on OF cues. The prototype Robot-Fly ("robot mouche") that we developed $[63,28]$ was a $50-\mathrm{cm}$ high, fully autonomous wheeled robot carrying a compound eye driving $114 \mathrm{OF}$ sensors with an omnidirectional azimuthal field of view (FOV). This reactive robot sensed the translational OF while moving straight ahead until detecting an obstacle, which triggered a quick eye and body turn of a suitable amplitude (during which time vision was inhibited). Since the Robot-Fly travelled at a constant speed $(50 \mathrm{~cm} / \mathrm{s})$, the OF measured during any translation easily gave the object range, and the robot was thus able to dodge and slalom to its target lamp through a random array of posts [28]. Despite the success of this early neuromimetic robot, flying insects would obviously have to use OF cues differently, since they are not in mechanical contact with the ground. How might flies, bees or micro aerial vehicles (MAVs) travel safely and cope with the many severe disturbances (obstacles, wind, etc.) they encounter?

Here we summarize our attempts to model the visuomotor control systems that provide flying insects with a means of close-range autonomous guidance. First we focus on ground avoidance in the vertical (longitudinal) plane (Section 3). We then discuss the ability to avoid corridor walls (Section 4), which has been closely analyzed in honeybees. Independent vertical and horizontal flight control systems (see also [62, 21]) were suggested by the performances of flies, which control their movements along the three orthogonal axes independently [98], while keeping their head transiently fixed in space [96, 100] via compensatory head roll and pitch mechanisms [42,43]. Experimental simulations were performed and our control schemes were tested on two fly-by-sight aerial robots: a micro-helicopter $(\mathrm{MH})$ (figure 5a) and a miniature hovercraft $(\mathrm{HO}$ ) (figure 8a). These aerial robots use neuromorphic OF sensors [7, 27, 8] inspired by the Elementary Motion Detectors (EMDs) previously studied at our laboratory in houseflies [70, 24, 29]. These sensors are briefly described in Section 2.

\section{From the fly EMDs to electronic optic flow sensors}

Conventional cameras produce images at a given frame rate. Each image is scanned line-by-line at a high frequency. Many authors working in the field of computer vision have presented algorithms for analyzing the OF field based on scanned camera images. Although an OF algorithm has been implemented onboard a highly miniaturized, slow but fully autonomous indoor MAV [102], none of the OF algorithms to be found in the insect brain actually start with a retinal image scanned line-by-line. Insects analyze the OF locally, pixel-by-pixel, via a neural circuit called an "Elementary Motion Detector" (EMD). Further down the neural pathways, well-known collator neurons called "lobula plate tangential cells" (LPTCs) integrate the outputs of large numbers of EMDs and analyze the OF field generated by the animal's locomotion. Some of them transmit electrical signals via the neck to thoracic interneurons directly or indirectly responsible for driving the wing, leg-, or head-muscles. Other LPTCs send relevant signals to the contralateral eye (Rev. [91, 39, 9, 94]).

To determine the functional principles underlying an EMD, the responses of an LPTC neuron were recorded $(\mathrm{H} 1$, figure $1 \mathrm{~b})$ while single photoreceptors in a single ommatidium were being stimulated using a high-precision instrument (a hybrid between a microscope and a telescope: figure 1d), where the main objective lens was a single ocular facet (diameter $\cong 25 \mu \mathrm{m}$, focal length $\cong 50 \mu \mathrm{m}$ ) (figure $1 \mathrm{a}$ ). By illuminating two neighboring photoreceptors successively, a motion occurring in the visual field of the selected ommatidium was simulated. The $\mathrm{H} 1$ neuron responded with a vigorous spike discharge to this "apparent motion", provided the motion was mimicked in the preferred direction (compare top and bottom traces in figure 1c) [70]. By applying various sequences of light steps and/or pulses to 
selected receptor pairs, an EMD block diagram was obtained and the dynamics and nonlinearity of each block were characterized [24, 29, 25].

In the mid 1980's, we designed a neuromorphic OF sensor based on the results of these electrophysiological studies [7, 27]. By definition, the OF is an angular speed $\omega$ corresponding to the inverse of the time $\Delta \boldsymbol{t}$ taken by a contrasting feature to travel between the visual axes of two adjacent photodiodes separated by an angle $\Delta \varphi$ (figure 2a). Our OF sensor's scheme is not a "correlator" [40, 68], but rather a "feature-matching scheme" [95], where a given feature (here, a passing edge) is extracted and tracked in time. Each photodiode signal is first band-pass filtered (figure 2b), mimicking the analog signals emitted by the large monopolar neurons present in the fly lamina [101]. The next processing step consists in performing hysteresis thresholding and generating a unit pulse. In the EMD version built in 1989 for the Robot-Fly (figure 2d), the unit pulse from one channel sampled a long-lived decaying exponential function generated by the other channel, via a nonlinear circuit called a minimum detector (figure 2b) giving a monotonically increasing output $V_{E M D}$ with the angular velocity $\omega=\Delta \varphi / \Delta t$ (figure $2 \mathrm{~b}$ ) [8]. The thresholding makes the EMD respond whatever the texture and contrast encountered, contrary to what occurs with "correlator" EMDs [68] (see also [21]).

A very similar EMD principle was developed independently a decade later by Koch's group at CALTECH, and termed the "facilitate and sample" velocity sensor [49]. These authors patented an aVLSI chip based on this principle [80], another variant of which was recently presented [57].

Our OF sensor actually comprises two parallel EMDs, each of which responds to either positive or negative contrast transitions, as in the fly EMD (cf figures 15\&16 in [29]). The circuit responds equally efficiently to natural scenes [64]. Our current OF sensors are still based on our original "travel time" principle $[7,27,8]$ but for the sake of miniaturization, the signals are processed using a mixed analog + digital approach [78] and the time $\Delta \boldsymbol{t}$ is converted into $\boldsymbol{\omega}$ via a look-up-table (figure 2c). Although they are much larger than any aVLSI (or fly's) EMDs, our current OF sensors (figure 2e,f) are small and light enough to be mounted on MAVs. Several OF sensors of this type can also be integrated into a miniature FPGA [1,2]. A different kind of OF sensor was recently designed and mounted on a model aircraft [6,37]. Optical mouse sensors have also been used as OF sensors [38, 13].

\section{An explicit control scheme for ground avoidance}

\subsection{Avoiding the ground by sensing the ventral optic flow}

The ventral OF perceived in the vertical plane by airborne creatures (including aircraft pilots) is the angular velocity $\omega$ generated by a point on the underlying flight track $[35,99]$. Based on the definition of the angular velocity (figure $3 a$ ), the ventral OF $\omega$ is the ratio between groundspeed $\boldsymbol{V}_{\boldsymbol{x}}$ and groundheight $\boldsymbol{h}$ :

$$
\omega=V_{x} / h\left[\mathrm{rad} . \mathrm{s}^{-1}\right]
$$

Flies and bees measure the translational $O F, \omega$, irrespective of the spatial texture and contrast encountered $[16,87,3]$, and some of their visual neurons respond monotonically to $\omega$ [47]. Neurons facing downwards can therefore act as ventral OF sensors and directly assess the groundspeed-togroundheight ratio $\boldsymbol{V}_{\boldsymbol{x}} / \boldsymbol{h}$ (figure 3 ).

Before Gibson introduced the OF concept [34], Kennedy established that an insect sees and reacts to the OF presented to its ventral viewfield [51] (see also [14]). This flowing visual contact with the ground is now known to be essential for insects to be able to orient upwind and migrate towards an attractive source of odour [51,50] or pheromones [53]. Based on field experiments on locusts, Kennedy developed the "optomotor theory" of flight, according to which locusts have a "preferred retinal velocity" with respect to the ground below [51,52]. In response to wind, for example, they adjust their groundspeed (or groundheight) to restore the velocity of the ground feature images. Kennedy's theory has been repeatedly confirmed during the last 30 years. Flies and bees seem to maintain a constant OF with respect to the ground while cruising or landing $[14,65,88,90,4]$.

The problem is how they achieve this remarkable feat, since maintaining a given OF is a kind of chicken-and-egg problem, as illustrated by Eq.1: an insect may hold its perceived OF, $\omega$ constant by controlling $\boldsymbol{V}_{\boldsymbol{x}}$ (if it knows $\boldsymbol{h}$ ) or by controlling $\boldsymbol{h}$ (if it knows $\boldsymbol{V}_{\boldsymbol{x}}$ ). Moreover, it could maintain an OF of say $1 \mathrm{rad} / \mathrm{s}$ (i.e., $57^{\circ} / \mathrm{s}$ ) by flying at a speed of $1 \mathrm{~m} / \mathrm{s}$ at a height of 1 meter or by flying at a speed of $2 \mathrm{~m} / \mathrm{s}$ at a height of $2 \mathrm{~m}$. There exists an infinitely large number of possible combinations of 
groundspeeds and groundheights generating the same "preferred OF"). Kennedy's "theory" therefore lacked an explicit control scheme elucidating:

1. the flight variables really involved

2. the sensors really required

3. the dynamics of the various system components

4. the causal and dynamic links between the sensor(s) and the variable(s) to be controlled

5. the points of application and the effects of the various disturbances that insects may experience

6 . the variables insects have to control to compensate for these disturbances.

Our first attempt to develop a control scheme was not very successful, as we were cornered by the chicken-and-egg OF problem mentioned above and by the assumption prevailing in those days that insect navigation involves estimating distance $[55,63,87,28,85]$. In the experimental simulations described in 1994, for example [58], we assumed that flying insects (and robots) must be able to measure their groundspeed $\boldsymbol{V}_{\boldsymbol{x}}$ (by whatever means), so that by measuring $\omega$ they would then be able to assess the distance $\boldsymbol{h}$ from the ground (Eq.1) and react accordingly to avoid it. Although this stratagem - which is in line with the Robot-Fly's working principles (Section 1) - may be acceptable for aerial vehicles that gauge their own groundspeed [5,33], it does not tell us how insects function.

In 1999, we established (via experimental simulations) how a seeing helicopter (or an insect) might manage to follow a terrain and land on the sole basis of OF cues without measuring its groundspeed or groundheight (see figures 4,5 in [60]). The landing maneuvers were performed under the permanent feedback control of an OF-sensing eye, and the driving force responsible for the loss of altitude was the decrease in the horizontal flight speed which occurred when the rotorcraft (or the insect) was about to land, either volontarily or because of an unfavourable headwind. The landing trajectory obtained in these simulations [60] resembled the final approach of bees landing on a flat surface [88]. The 840-gram rotorcraft we constructed was able to jump over 1-meter high obstacles (see figure 8 in [61]).

\subsection{The "Optic flow regulator"}

We recently developed a genuine "OF based autopilot" called OCTAVE (which stands for Optical altitude Control sysTem for Aerial VEhicles), that enables a micro-helicopter to perform challenging tasks such as take-off, terrain following, reacting suitably to wind, and landing [73-77]. The idea was to integrate an OF sensor into a feedback loop driving the robot's lift so as to compensate for any deviations of the OF sensor's output from a given set-point. This is what we call the OF regulator for ground avoidance. The term 'regulator' is used here as in control theory, to denote a feedback control system designed to maintain a variable constantly equal to a given set-point. The variable regulated is often a temperature, a speed, or a distance, but here it is the variable $\omega[\mathrm{rad} / \mathrm{s}] \mathrm{corresponding}$ to the $\boldsymbol{V}_{\boldsymbol{x}}: \boldsymbol{h}$ ratio, which can be sensed directly by an OF sensor. The OF sensor produces a signal $\boldsymbol{\omega}_{\text {meas }}$ (figures $3 b$ ) that is compared with an OF set-point $\omega_{\text {Set }}$ (figure 4a). The error signal $\varepsilon=\omega_{\text {meas }}-\omega_{\text {Set }}$ drives a controller adjusting the lift $\boldsymbol{L}$, and hence the groundheight $\boldsymbol{h}$, so as to minimize $\boldsymbol{\varepsilon}$. All the operator does is to set the pitch angle $\Theta$ and hence the forward thrust, and hence the airspeed (see figure $4 a$ ): the OF regulator does the rest, keeping the OF, i.e., the $\boldsymbol{V}_{\boldsymbol{x}}: \boldsymbol{h}$ ratio, constant. In the steady state (i.e., at $\boldsymbol{t}=\infty), \boldsymbol{\omega}_{\text {meas }} \cong \omega_{\text {Set }}$ and the groundheight $\boldsymbol{h}$ becomes proportional to the groundspeed $\boldsymbol{V}_{\boldsymbol{x}}($ Eq.2):

$$
h=K V_{x} \quad\left(\text { with } K=1 / \omega_{\mathrm{SET}}=\text { constant }\right)
$$

\subsection{Micro-helicopter (MH) with a downward-looking optic flow sensing eye}

To test the robustness of the OF regulator, we implemented it on a micro-helicopter (MH) equipped with a ventral OF sensor [73]. The robot (figure 5a) is tethered to the arm of a flight mill driven in terms of its elevation and azimuth by the MH's lift and forward thrust, respectively (figure 5b). Any increase in the rotor speed causes the $\mathrm{MH}$ to lift and rise, and the slightest (operator mediated) forward tilting induces the $\mathrm{MH}$ to gain speed. The flight mill is equipped with ground-truth azimuthal and elevation sensors with which the position and speed of the MH can be measured with great accuracy in real time. The MH is equipped with a minimalistic two-pixel ventral eye driving a single EMD (as in figure $3 e)$. The latter senses the OF produced by the underlying arena covered by a richly textured, randomly distributed pattern in terms of both the spatial frequency and the contrast $\boldsymbol{m}(0.04<\boldsymbol{m}<0.3)$. 
Whenever the robot is required to pitch forwards or backwards, the eye is actively counterrotated by a micro-servo that keeps the gaze oriented vertically downwards. This process mimicks the pitch stabilization mechanism at work in the eye in flying flies, ensuring suitable alignment of the eyes in space [43]. It eliminates the adverse effects of the rotational OF and the resulting need for a "derotation" procedure $[86,102]$.

\section{4. insects' versus the seeing helicopter's behavioral patterns}

OCTAVE's OF regulator scheme (figure 4a) results in the behavioral patterns shown in figure 6 , which gives the $\mathrm{MH}$ flight variables monitored during a 70-meter flight over a flat terrain [30]. In figure 6a (left), the operator simply pitched the $\mathrm{MH}$ forward rampwise by an angle $\Delta \Theta=+10^{\circ}$ (between arrowheads 1 and 2). The ensuing increase in groundspeed $V_{\boldsymbol{x}}$ (up to $3 \mathrm{~m} / \mathrm{s}$, see $b$ ) automatically made the $\mathrm{MH}$ take off, since the feedback loop consistently increased $\boldsymbol{h}$ proportionally to $\boldsymbol{V}_{\boldsymbol{x}}$ to comply with $E q$. 2. The MH eventually flew level at a groundheight $\boldsymbol{h}$ of approximately 1 meter - the value imposed by the OF set-point $\omega_{\text {set }}$ (figure 6c). After covering 42 meters, the $\mathrm{MH}$ was pitched backwards rampwise by an opposite angle $\Delta \Theta=-10^{\circ}$ (between arrowheads 3 and 4), and the ensuing deceleration (see $b$ ) automatically triggered a gradual descent until landing occurred. As the landing gear kept the robot's eye 0.3 meter above ground (dotted horizontal line), touchdown occurred shortly before the groundspeed $\boldsymbol{V}_{\boldsymbol{x}}$ had reached zero, and the MH ended its journey with a short ground run.

The MH flight pattern shows that an airborne vehicle can take off, navigate and even land on flat terrain without having to measure the groundheight or groundspeed, provided it is equipped with an OF sensor facing the ground and an OF regulator.

The OF regulator concept and the robot's performances - which are extremely reproducible [76] - were found to account for a series of puzzling, seemingly unconnected flying abilities observed by many authors during the last 70 years in various species (fruitflies, honeybees, moths, mosquitoes, dungbeetles, migrating locusts, butterflies and birds), as discussed in [30]. The most striking parallels with insect behavior focus on:

- automatic terrain following. A gradual increase in relief constitutes a "disturbance" that impinges on the system at a particular point (see figure 4a). The closed feedback loop overcomes this perturbation by increasing the flight altitude, resulting in a constant groundheight over the rising terrain [73]. This may account for the terrain and canopy following abilities of migrating insects, as described in [30].

- suitable reactions to headwind. Windspeed is a disturbance that impinges on the system at a different point (see figure 4a). The feedback loop overcomes this perturbation by forcing the robot to descend (and even to land smoothly by strong wind; see figure 8 in [74]). A similar reaction was observed in locusts, honeybees and dung beetles, as reported in [30].

- flight over a no-contrast zone. Here the OF sensor fails to respond, which irremediably causes the robot to crash, just as honeybees crash into mirror-smooth water, as observed in 1963 [44].

- landing on a flat surface. During the final approach, which starts when the $\mathrm{MH}$ has regained its completely upright position (arrowhead 4 in figure 6a), the OF regulator forces the MH to land smoothly at a constant descent angle, $\boldsymbol{\alpha}$ [rad] (figure 6a), which depends on only two parameters (Eq.3):

$$
\alpha=-\arctan \left(\frac{1}{\omega_{\text {set }} \tau}\right)
$$

where $\omega_{\text {set }}\left[\right.$ rad.s $\left.{ }^{-1}\right]$ is the OF set-point, and $\tau$ [s] is the surge time constant of the MH $\left(\tau_{M H}=2.15 \mathrm{~s}\right)$ [30].

Honeybees also land with a constant slope on flat surfaces [90]. The way bees may achieve this feat has been explained quite differently, however. Landing bees would follow two rules: "(1) adjusting the speed of forward flight to hold constant the angular velocity of the image of the surface as seen by the eye, and (2) making the speed of descent proportional to the forward speed" [90]. By contrast, our OF regulator automatically generates smooth landing with a constant slope without either the forward speed or the descent speed being controlled (details in [30]).

In short, the MH's outstanding visuo-motor performances and their close resemblance to insects' behavioral patterns show how insects and MAVs may take off, follow terrain and even land smoothly without having to measure the groundheight, groundspeed or descent speed, provided they are equipped with OF sensors facing the ground and an OF regulator that servoes the OF to a reference value. 
This model differs markedly from another one where the OF controls the groundspeed $\boldsymbol{V}_{\boldsymbol{x}}$ rather than the groundheight $\boldsymbol{h}[66,90,3,88]$. It can be seen in figure 4 a that if the ventral OF controlled $\boldsymbol{V}_{\boldsymbol{x}}$ instead of $\boldsymbol{h}$, this would generate strikingly different flight patterns, as follows:

(i) instead of following a slanting terrain, as migrating butterflies and our $\mathrm{MH}$ do, insects would gradually decelerate until touching the rising ground at a negligible speed, thus inopportunely interrupting their journey.

(ii) instead of descending in a headwind and rising in a tailwind, as honeybees [e.g. 71], locusts [52] and our $\mathrm{MH}$ do, insects would compensate for an unfavourable headwind by increasing their airspeed without changing their groundheight.

These two models can be reconciled, however, if we add the hypothesis that another OF regulator based on OF sensors oriented towards the lateral parts of the eyes could be used to control the groundspeed. In the next Section, we give the example of flight in a corridor to show how these lateral OFs might control the insect's behavior by determining both the groundspeed and the clearance from the walls.

\section{An explicit control scheme for speed control and lateral obstacle avoidance}

Based on behavioral experiments on honeybees conducted at Srinivasan's laboratory and at our own laboratory, we then designed the LORA III autopilot (LORA III stands for Lateral Optic flow Regulator Autopilot, mark III), which is able to control both the forward speed $\boldsymbol{V}_{\boldsymbol{x}}$ of an aerial vehicle and its lateral distances $\boldsymbol{D}_{\boldsymbol{r}}$ and $\boldsymbol{D}_{\boldsymbol{I}}$ from two corridor walls [82].

\subsection{Effects of lateral OF on wall clearance and forward speed}

Honeybees flying through a narrow corridor tend to fly along the midline [55, 87]. To explain this "centering behavior", the authors hypothesized that bees might balance the speeds of the retinal images (i.e., the lateral OFs) of the two walls. This was confirmed by experiments where one wall was set in motion: bees flying in the same direction as the moving wall tended to fly closer to it, whereas bees flying in the opposite direction tended to fly farther away from it. These experiments showed compellingly that the visuomotor control mechanism at work in flying bees depends on the laterally perceived translational OF [87].

We recently reported that honeybees trained to fly along a corridor toward a nectar source did not systematically center on the corridor midline, but could hug one wall (figure $7 b, c$ ) [83]. Bees kept on hugging one wall even when part of the opposite wall was missing (figure 7d). Interestingly, forward speed $V_{x}$ and distances from the walls, $D_{R}$ and $D_{L}$, were on average such that the speed-to-distance ratio (i.e., the lateral OF, $\omega$ ) was maintained virtually constant (at about $230 \%$ in our $95-\mathrm{cm}$ wide corridor) [83]. These novel findings prompted us to examine how bees may adopt either "centering behavior" (figure 7a) or "wall-following" behavior (figure 7b-d) on the basis of OF-sensing, a prowess that seems to require jointly controlling the forward speed and the clearance from the walls.

Experiments on tethered $[36,10]$ and free-flying flies [15-17] and bees [88, 3] and tethered locusts [84] have long shown that motion detected in the lateral part of these insects' compound eyes affects the forward thrust, and hence the forward speed. When flying through a tapered corridor lined with regular black and white vertical stripes, honeybees slowed down as they approached the narrowest section and speeded up when the corridor widened beyond this point [88]. Bees therefore tended to adjust their speed proportionally to the local corridor width. The authors concluded that "the speed of the flight is controlled by regulating the image velocity" [88].

The honeybee's "centering behavior" was successfully mimicked by numerous terrestrial robots [e.g.,12, 19, 79, 89]. The speed of one of the early robots was also controlled on the basis of the sum of the lateral OFs perceived on both sides [79].

\subsection{New robotic demonstrator based on a hovercraft $(\mathrm{HO})$}

With a view to explaining honeybees' behavior in a corridor, a miniature hovercraft $(\mathrm{HO})$ was used [82]. With an aerial vehicle of this kind endowed with natural roll and pitch stabilization abilities, planar flight control systems can be developed conveniently. Like flies [98, 96] and sandwasps [100], our 
modified $\mathrm{HO}$ can produce forward and sideward slips independently, because the two lateral thrusters $L T 1$ and $L T 2$ that we have added (figure 8) make it fully actuated. It travels at a constant altitude $(\sim 2 \mathrm{~mm})$ and senses the environment with two laterally oriented eyes, which measure the right and left OFs. Each eye contains only two photoreceptors, each driving an EMD of the type shown in figure 2e. The HO's heading is maintained along the X-axis of the corridor (figure 8b) by a heading lock system (based on a micro-magnetic compass enhanced by a rate-gyro), which compensates for any yaw disturbances by controlling the two rear thrusters differentially. This system mimicks the honeybee's heading lock system, which is based on a polarized light compass [32, 72] and gives the insect an impressively straight course even in the presence of wind [71].

The 820-gram HO travels at a groundspeed vector $\vec{V}$ over a flat surface along a corridor, the walls of which are wall-papered with vertical stripes with a random pattern of spatial frequency and contrast, mimicking a richly textured visual environment (figure $8 \mathrm{~b}$ ). Since any yaw rotations are compensated for, each eye experiences a purely translational OF, $\omega_{R}$ or $\omega_{L}$, which can be simply defined as follows:

$$
\begin{aligned}
& \omega_{R}=V_{x} / D_{R} \\
& \omega_{L}=V_{x} / D_{L}
\end{aligned}
$$

where $V_{x}$ is the hovercraft's groundspeed, $D_{R}$ and $D_{L}$ are the distances from the right and the left walls, respectively.

\subsection{The LORA III autopilot: a dual OF regulator}

The hovercraft is controlled by the bio-inspired autopilot called LORA III (figure 9) [82]). This control scheme has been called the dual OF regulator, because it consists of two strongly interdependent feedback loops, the side control loop and the forward control loop, each of which uses the data collected by the OF sensors looking to both sides. Each loop controls the thrust along its own translational degree of freedom (i.e., in the surge or sway direction) and has its own OF set-point. The tight coupling existing between the two loops is illustrated by the fact that the feedforward gain in the side control loop is proportional to the forward speed $V_{x}$, which is determined in turn by the forward control loop (see figure 9). The hovercraft reacts to any changes in the lateral OFs by selectively adjusting the two orthogonal components $\boldsymbol{V}_{\boldsymbol{x}}$ and $\boldsymbol{V}_{\boldsymbol{y}}$ of its groundspeed vector $\vec{V}$ according to the following two principles:

\section{Side control system}

The principle underlying the side control system (figure 9, bottom loop) is based on findings made on the honeybee's centering and wall-following behavior described in 4.1. The side control system is a lateral OF regulator akin to the ventral OF regulator for ground avoidance (OCTAVE: see Section 3). It takes the OF generated by the two corridor walls into account, and strives to keep the larger of the two OFs measured (left or right) constantly equal to a sideways OF set-point $\omega_{\text {setside. }}$. The hovercraft then reacts to any OF deviations from $\omega_{\text {Setside }}$ by controlling the lateral thrust, and hence the side slip speed $V_{y}$, and hence the distance from the left or right wall. A maximum criterion selects the larger of the two OFs measured, $\omega_{\text {Rmeas }}$ or $\omega_{\text {Lmeas }}$, and the sign of their difference determines which wall will be followed. The larger OF is compared with the sideways OF set-point $\omega_{\text {Setside }}$.The error signal $\boldsymbol{\varepsilon}_{\text {side }}$ transmitted to the side controller (figure 9) is therefore calculated as follows :

$$
\varepsilon_{\text {side }}=\operatorname{sign}\left(\omega_{\text {Lmeas }}-\omega_{\text {Rmeas }}\right) \times\left(\omega_{\text {SetSide }}-\max \left(\omega_{\text {Lmeas }}, \omega_{\text {Rmeas }}\right)\right)
$$

In the steady state, the OF selected will become equal to $\omega_{\text {Setside }}$ due to the natural integrator present in this loop. The side-control loop is nonlinear since the OF is an inverse function of the variable $D_{L}$ or $D_{R}$ controlled. The transfer function of the "side dynamics" (figure 9) relating the hovercraft's side speed $\boldsymbol{V}_{\boldsymbol{y}}$ to the control signal $\left(\boldsymbol{u}_{L T 1}-\boldsymbol{U}_{L T 2}\right)$ delivered by the side controller approximates a first-order low-pass filter with an identified time constant of 0.5s [82]. A proportional-derivative (PD) controller was introduced into the side controller to increase the damping, thus improving the stability and enhancing the response dynamics. Details of the tuning procedures are given in [82]. 
The forward control system (figure 9, upper loop) is based on the OF perceived on both sides, as reported in 4.1 for a robot [79] and for the honeybee [88]. This control system strives to hold the sum of the left and right OFs measured $\left(\omega_{\text {Lmeas }}+\omega_{\text {Rmeas }}\right)$ constant and equal to a forward OF set-point $\omega_{\text {SetFwd }}$ by controlling the forward thrust, and hence the forward speed $V_{x}$. This control scheme automatically generates a "safe" forward speed, i.e., one commensurate with the local corridor width. More specifically, the feedback signal corresponding to the sum of the two OFs measured is compared with the forward OF set-point $\omega_{\text {SetFwd }}$ (figure 9 ). In the steady state, the sum of the OF becomes equal to $\omega_{\text {SetFwd }}$.

The transfer function of the "forward dynamics" relating the hovercraft's forward speed $\boldsymbol{V}_{\boldsymbol{y}}$ to the forward control signal $u_{R T 1}+u_{R T 2}$ (figure 9) is also given by a first order low-pass filter with a time constant of $0.5 \mathrm{~s}$ [82]. A proportional-integral (PI) controller was introduced here into the feedback loop to improve the closed-loop dynamics and give a zero steady state error. Futher details of the tuning procedures used are given in [82].

\section{4. "Operating point" of the dual OF regulator}

The scheme in figure 9 requires neither the speed nor the distance from the walls to be measured. The question therefore arises as to whether the system will converge and give a single steady state "operating point" in terms of a specific forward speed and a specific distance from the walls. In the following discussion, the LORA III autopilot is assumed to have reached the steady state $(t=\infty)$. That is, (i) the sum of the two lateral OFs measured, $\left(\omega_{\text {Rmeas }}+\omega_{\text {Lmeas }}\right)$ has reached the forward OF set-point $\omega_{\text {SetFwd }}$, and (ii) the larger of the two lateral OFs measured (here it is taken to be the right one, $\omega_{\text {Rmeas }}$ ) has reached the sideways OF set-point $\omega_{\text {setside }}($ see above). We can therefore write:

$\left\{\begin{array}{l}\omega_{\text {SetFwd }}=\omega_{R \infty}+\omega_{L \infty} \\ \omega_{\text {SetSide }}=\omega_{R \infty}\end{array} \Leftrightarrow\left\{\begin{array}{l}\omega_{\text {SetFwd }}=\frac{V_{x \infty}}{D_{R \infty}}+\frac{V_{x \infty}}{D_{L \infty}} \\ \omega_{\text {SetSide }}=\frac{V_{x \infty}}{D_{R \infty}}\end{array}\right.\right.$

The forward speed $\boldsymbol{V}_{x^{\infty}}$ and the ordinate $\boldsymbol{y}_{\infty}=D_{R}$ that the hovercraft will reach in the steady state can be said to define an "operating point" $\left(\boldsymbol{V}_{\boldsymbol{x}_{\infty}}, \boldsymbol{y}_{\infty}\right)$ that can be calculated straightforwardly from Eqs.7 and 8 as follows:

$$
\left\{\begin{array}{c}
V_{x \infty}=\frac{\omega_{\text {SetSide }} \cdot\left(\omega_{\text {SetFwd }}-\omega_{\text {SetSide }}\right)}{\omega_{\text {SetFwd }}} \cdot D \\
y_{\infty}=D_{R \infty}=\frac{\omega_{\text {SetFwd }}-\omega_{\text {SetSide }}}{\omega_{\text {SetFwd }}} \cdot D, \quad y_{\infty} \in\left[0, \frac{D}{2}[\right. \\
D=D_{R \infty}+D_{L \infty}, \text { where } D \text { is the corridor width }
\end{array}\right.
$$

Equations 9, 10 have four important consequences:

- Two single parameters, $\omega_{\text {SetFwd }}$ and $\omega_{\text {SetSide }}$, suffice to define the speed and lateral position $\left(\boldsymbol{V}_{\boldsymbol{x}_{\infty},}, \boldsymbol{y}_{\infty}\right)$ that the hovercraft (or the bee) will adopt in a corridor of a given width $\boldsymbol{D}$ : the flying agent needs to measure neither its speed nor its clearance from the walls nor the corridor width.

- Both the speed $\boldsymbol{V}_{x_{\infty}}$ and the ordinate $\boldsymbol{y}_{\infty}$ adopted in the steady state can be seen to be proportional to the corridor width $\boldsymbol{D}$ (Eqs. 9,10). If the corridor is twice as large, the agent will 
automatically fly twice as fast. This mechanism therefore accounts for the fact that "the bee controls its flight speed by monitoring and regulating the angular velocity of the environment's image as represented on the eye; that is, if the tunnel width is doubled, the bee flies twice as fast" [88]. From Eq. 10, it can be said that in this case the clearance from the wall followed will be twice as large even when the bee does not fly centered (as in figures $7 b-d$ ).

- If the sideways OF set-point is larger than half the value of the forward OF set-point (i.e., $\left.w_{\text {SetSide }}>w_{\text {SetFwd }} / 2\right)$, the agent will reach a final ordinate $y_{\infty}(E q .10)$ that is smaller than half the value of the corridor width. This means that the flying agent will hug one wall, thus generating wall-following behavior.

- At all sideways OF set-point values that are smaller than half the forward OF set-point (i.e., $\omega_{\text {SetSide }}<\omega_{\text {SetFwd }} / 2$ ), the agent will not reach the final ordinate $\boldsymbol{y}_{\infty}$ predicted by $E q .10$ without causing a change in sign in the error signal $\varepsilon_{\text {side }}$ (see Eq. 6). The agent will therefore be forced to maintain the OF value at $\omega_{\text {SetFwd }} / 2$, i.e., it will consistently navigate along the midline of the corridor, thus showing centering behavior. Oscillations in the trajectory are likely to occur, however, because of the repeatedly changing sign of the error signal $\boldsymbol{\varepsilon}_{\text {side }}(E q .6)$.

\subsection{Simulation results: flight paths along straight or tapered corridors}

The implementation of the complete LORA III visuo-motor autopilot on the robotic hovercraft is underway, and only computer-simulated experiments will be presented here [82]. The computer simulations include: the dynamic hovercraft model on both the surge and sway axes, actuator saturations, full optical transfer function of the lens/photoreceptor system of each eye, detailed interactions between each photoreceptor and the random patterns lining the two walls, and complete processing within each OF sensor, according to the EMD principle described in figure 2c. These simulations were carried out on a standard PC equipped with the Matlab ${ }^{\mathrm{TM}} /$ Simulink toolbox at $a$ sampling frequency of $1 \mathrm{kHz}$. This sampling frequency is the same as the sampling rate of the microcontroller installed onboard the LORA robot (figure 8a).

\section{Wall-following behavior along a straight corridor}

Figures 10-12 show some examples of the computer-simulated behavior of the hovercraft equipped with the LORA III autopilot (figure 9) [82]. The first visual environment simulated was a straight corridor ( 3 meters long, 1 meter wide). The right and left walls were lined with a random pattern of various grey vertical stripes covering a 1-decade contrast range (from $4 \%$ to $38 \%$ ) and a 1.1-decade spatial frequency range (from $0.068 \mathrm{c} /{ }^{\circ}$ to $0.87 \mathrm{c} /{ }^{\circ}$ reading from the corridor midline). From the three simulated trajectories shown in figure $10 a$, the hovercraft can be seen to navigate without ever colliding with the walls. It reached the same steady speed regardless of its initial ordinate $y_{0}$ at the entrance to the corridor. All three trajectories corresponded to the case where the sideways OF set-point was larger than half the forward OF set-point $\left(\omega_{\text {Setside }}=230 \%>150 \%=\omega_{\text {SetFwd }} / 2\right)$. Under these conditions, the $\mathrm{HO}$ adopted wall-following behavior, as predicted in Section 4, and followed the right or left wall, depending on the initial ordinate $y_{0}$. The $\mathrm{HO}$ consistently generated a steady state clearance of $0.25 \mathrm{~m}$ from either wall (left wall: squares, and crosses; right wall: full dots) and a "safe" forward speed of $V_{x \infty}=1 \mathrm{~m} / \mathrm{s}$. Steady state clearance and speed define a similar "operating point" to that calculated from Eqs. 9,10: taking $\omega_{\text {SetFwd }}=300 \%, \omega_{\text {SetSide }}=230 \%$ and a corridor width $D=1 \mathrm{~m}$ gives an operating point $V_{x^{\infty}}=0.94 \mathrm{~m} / \mathrm{s}$ and $y_{\infty}=0.23 \mathrm{~m}$.

Whether the hovercraft followed the right or the left wall depended on the sign of the error signal $\varepsilon_{\text {side }}$ (Eq. 6). The hovercraft's initial ordinate $y_{0}$ was treated like a disturbance (see figure 9), which was rejected by the dual OF regulator. It can be seen from figure $10 \mathrm{~d}$, f that both the sum and the larger value of the lateral OFs equal the OF set-points of $300 \%$ and $230 \%$, respectively.

\section{"Centering behavior" : a particular case of "wall-following behavior"}

Figure 11 illustrates the opposite case, where the OFs generated on either side $\left(150^{\circ} / \mathrm{s}\right.$ : figure $\left.11 \mathrm{~d}\right)$ never reached the sideways OF set-point OF, $\omega_{\text {Setside }}$ (which was set at $90 \%$, or $110 \%$ or $130 \%$ s) because these required values of $\omega_{\text {Setside }}$ were all smaller than half the value of the forward OF setpoint (i.e., $\omega_{\text {SetSide }}<\omega_{\text {SetFwd }} / 2=150^{\circ} / \mathrm{s}$ ). These low values of $\omega_{\text {SetSide }}$ relative to $\omega_{\text {SetFwd }}$ forced the hovercraft to center between the two walls, as predicted at the end of Section 4.4. In addition, the 
robot's ordinate can be seen to oscillate about the midline, due to the ever-changing sign of the error signal $\varepsilon_{\text {side }}(E q .6)$ - as also predicted at the end of Section 4.4. Thus, the striking oscillatory flight pattern observed in honeybees flying in a centered position along a corridor (see figure 2a in $[55,87]$ ) is nicely accounted for by the LORA III dual OF regulator. The error signal $\varepsilon_{\text {side }}$ is consistently minimum along the midline, but the OF cannot become smaller than $150^{\circ} / \mathrm{s}$ (i.e., $\omega_{\text {SetFwd }} / 2$ ). The hovercraft can be seen to have reached the steady state forward speed of $V_{x^{\infty}}=1.3 \mathrm{~m} / \mathrm{s}$ (Fig. 11b) in all three cases because all three values of $\omega_{\text {Setside }}$ were below $150 \%$. In all three cases, the steady state operating point of the hovercraft was similar to that predicted in Section 4.4: at $\omega_{\text {SetFwd }}=300 \%$ and $\omega_{\text {Setside }}<150 \%$, a 1 -meter wide corridor gives: $V_{x^{\infty}}=1.31 \mathrm{~m} / \mathrm{s}$ and $y_{\infty}=D_{R_{\infty}}=D_{L_{\infty}}=0.5 \mathrm{~m}$ (Eqs. 9 \& 10). The lateral OFs measured on either side reached $150 \%\left(=\omega_{\text {SetFwd }} / 2\right)$ in all three cases (Fig 11c), and their sum was therefore equal to $300 \% \mathrm{~s}\left(=\omega_{\text {SetFwd }}\right)$. Similar centering behavior occurred at all values of $\omega_{\text {Setside }}$ such that $\omega_{\text {SetSide }} \leq \omega_{\text {SetFwd }} / 2$. Centering behavior can therefore be said to be a particular case of wall-following behavior.

\section{Flight pattern along a tapered corridor}

In the second set of computer-simulated experiments presented here, the environment was a 6meter long tapered corridor with a 1.24-m wide entrance and a $0.5-\mathrm{m}$ wide constriction located midway (figure 12). The right and left walls were lined with a random pattern of grey vertical stripes covering a 1-decade contrast range (from $4 \%$ to $38 \%$ ), and a 1.5-decade spatial frequency range (from $0.034 \mathrm{c} /{ }^{\circ}$ to $1.08 \mathrm{c} /{ }^{\circ}$ reading from the corridor midline).

As shown in Figure 12a, whatever the position of its initial ordinate $y_{0}$, the $\mathrm{HO}$ automatically slowed down on approaching the narrowest section of the corridor and accelerated when the corridor widened beyond this point. In figure 12a, the $\mathrm{HO}$ adopted wall-following behavior because $\omega_{\text {Setside }}>\omega_{\text {SetFwd }} / 2=$ $150 \%$ s (see Eq. 10). It can be seen from Figure 12c that the forward control system succeeded in keeping the sum of the two lateral OFs measured constant and equal to the forward OF set-point $\omega_{\text {SetFwd }}=300 \%$. Likewise, the side control system succeeded in keeping the larger of the two lateral OFs measured virtually constant and equal to the sideways OF set-point $\omega_{\text {Setside }}=230 \%$ (figure $12 \mathrm{e}$ ). Not only the initial ordinate $y_{0}$ but also the (gradually changing) ordinates, $y_{R}$ and $y_{L}$, are regarded by the LORA III autopilot as output perturbations, which are rejected by the dual OF regulator (see the points of application of these disturbances in figure 9).

The ensuing forward speed profile along the tapered corridor is particularly instructive (figure 12b): the HO's forward speed $\boldsymbol{V}_{\boldsymbol{x}}$ tends at all times to be proportional to the distance travelled $\boldsymbol{x}$, as observed with the flight path of bees flying freely along a tapered corridor [88]. This plot of $\boldsymbol{V}_{\boldsymbol{x}}=\mathbf{d x} / \mathbf{d t}$ versus $\mathbf{x}$ actually defines a phase plane, in which the linear change in speed observed with the distance travelled means that the speed $\boldsymbol{V}_{\boldsymbol{x}}(t)$ is bound to vary as an exponential function of time [82]:

$V_{x}(t)=V_{x}\left(t_{0}\right) \cdot \mathrm{e}^{\operatorname{sign}(\alpha) \cdot\left(t-t_{0}\right) / \tau(\alpha)}$

with a time constant $\tau(\alpha)$ which is a monotonic function of the tapering angle $\alpha$, as follows:

$$
\tau(\alpha)=\frac{\omega_{\text {SetFwd }}}{2 \cdot \tan |\alpha| \cdot \omega_{\text {SetSide }} \cdot\left(\omega_{\text {SetFwd }}-\omega_{\text {SetSide }}\right)}
$$

Without having any knowledge of the corridor width $\boldsymbol{D}$ or the tapering angle $\alpha$, the $\mathrm{HO}$ (or the bee) is therefore bound to slow down exponentially with a time constant $\tau(\alpha)$ when entering the narrowing section $(\alpha<0)$ of the corridor and to speed up exponentially with the same time constant $\tau(\alpha)$ after leaving the constriction $(\alpha>0)$.

The behavioral effects of two other types of disturbance affecting the LORA III autopilot were also studied [82]. With a large gap in a wall (as in the bee experiments shown in figure $7 \mathrm{~d}$ ) the HO was not flummoxed and kept on following the remaining wall [82]. When a wall pattern was moved at a constant speed $V_{P}$ in the direction of travel (as in the original bee experiments [55]), the robot moved closer to the moving wall, and vice versa [82]. Under these conditions, the robot's relative speed with respect to the moving wall became $\boldsymbol{V}_{\boldsymbol{x}}-\boldsymbol{V}_{\boldsymbol{P}}$ instead of $\boldsymbol{V}_{\boldsymbol{x}}$ in Eqs. 7,8, causing a predictable shift in the robot's operating point, $\boldsymbol{V}_{\boldsymbol{x} \infty}, \boldsymbol{y}_{\infty}$ as computed from Eqs. 9, 10. 


\section{Conclusion}

\subsection{Is there a pilot onboard an insect?}

In this chapter, we have recounted our attempts to specify the types of operations that insects may perform to guide their flight on the basis of optic flow (OF) cues. The OCTAVE principle differs markedly from another current OF based navigation strategy, where OF sensing needs to be completed by groundspeed sensing (based on a GPS, for example) to estimate the groundheight (see Eq.1) so as to follow terrain and land safely [5, 33]. The OCTAVE and LORA III autopilots harness the power of the translational OF more parsimoniously because they do not need to measure or estimate any distances or groundspeeds, and do not require any sensors other than OF sensors. The purpose of these autopilots is not to regulate any distances or groundspeeds. The only variable they need to regulate (i.e., maintain constant) is the OF - a variable which represents a speed-to-distance ratio that can be directly measured by a dedicated sensor called an OF sensor. OCTAVE and LORA III autopilots include three interdependent OF regulators in all, which control the lift, lateral thrust, and forward thrust, on which the groundheight, lateral positioning and groundspeed, respectively, depend. The block diagrams (Figures $4 \& 9$ ) show which variables need to be measured, which ones are controlled and which ones are regulated, as well as the point of application of the various disturbances. They also give the causal and dynamic relationships between these variables. These three feedback control loops may enable an agent having no mechanical contact with the ground to automatically attain a given groundspeed, a given groundheight and a given clearance from the walls in a simple environment such as a corridor, without any need for speed or range sensors giving explicit speed or distance data. In a tapered corridor, for example, the hovercraft $(\mathrm{HO})$ automatically tunes both its clearance from the walls and its groundspeed to the local corridor width (figure 12), although it is completely "unaware" of the exact corridor width, the forward speed and the clearance from the walls. The behavior depends wholly on three parameters, which are the three OF setpoints $\omega_{\text {SetVentr }}, \omega_{\text {SetSide }}$ and $\omega_{\text {SetFwd. }}$. Experimental simulations and physical demonstrators showed that difficult operations such as automatic takeoff, ground avoidance, terrain following, centering, wallfollowing, suitable reaction to headwind, groundspeed control and landing, can be successfully performed on the basis of these three OF regulators.

These control schemes account for many surprising findings published during the last 70 years on insects' visually guided performances (details in [30]) including honeybees' habit of landing at a constant slope [90] and their flight pattern along a tapered corridor [88]. Our novel finding that bees do not center systematically in a corridor but tend to follow a wall, even when the opposite wall has been removed (figure $7 \mathrm{~b}-\mathrm{d}$ ), cannot be accounted for by the optic flow balance hypothesis $[55,87]$ and is convincingly accounted for by the LORA III model, where "centering behavior" $[55,87]$ turned out to be a particular case of "wall-following behavior" (Section 4.5). LORA III (figure 9) would give the bee a safe speed and safe clearance from the walls, whereas OCTAVE (figure 4) would give the bee safe clearance from the ground - a clearance commensurate with its forward speed, whatever the speed. These explicit control schemes can therefore be viewed as working hypotheses. Onboard insects, the three OF set-points may depend on either innate, internal or external parameters. Recent findings have shown that the forward speed of bees flying along a corridor depends not only on the lateral OF but also partly on the ventral OF [4], which suggests that the ventral and lateral OFs should not perhaps be handled as separately as with OCTAVE and LORA III. Recent experimental simulations have shown how an agent might fly through a tunnel by relying on the OF generated by all four sides: the lateral walls, ground and roof [64]. Indoor experiments on the autonomously flying microplane MC2 showed that when the plane makes a banked turn to avoid a wall, the ventral OF may originate partly from the lateral wall, and the lateral OF partly from the ground [102]. This particularity may not concern insects, however, since they compensate for the banking and pitching of their thorax $[42,43]$ by actively stabilizing their "visual platforms" [96, 100]. A high-speed, one-axis oculomotor compensatory mechanism of this kind was recently implemented onboard a 100-gram aerial robot [54].

The electronic implementation of an OF regulator is not very demanding (nor is its neural implementation), since it requires only a few linear operations (such as adding, subtracting and applying various filters) and nonlinear operations (such as minimum and maximum detection). OF sensors are the crux of OF regulators. Our neuromorphic OF sensors deliver an output that grows monotonically with the OF $\omega$, regardless of the spatial frequency and contrast encountered (see Section 2), much like honeybees' velocity tuned (VT) neurons [47]. An OF regulator scheme greatly reduces the dynamic range constraints imposed on OF sensors, since it tunes the animal's behavior so that the OF will deviate little from the OF set-point [30]. In figure 6c, for example, the MH holds 
$\omega_{\text {meas }}$ virtually constant throughout its journey - even during takeoff and landing, when large groundspeed variations occur (see figure 6b). This and other examples (see figures 10c,e; 11c and $12 \mathrm{c}, \mathrm{e}$ ) show that the one-decade dynamic range (from $40^{\circ} \mathrm{s}$ to $400^{\circ} / \mathrm{s}$ [78]) of our OF sensors is up to the tasks.

\subsection{Potential aeronautics and aerospace applications}

The control schemes presented here rely on the OF to carry out reputedly difficult tasks such as taking off, terrain following, landing, avoiding lateral walls and groundspeed control. These simple control schemes are restricted so far to cases where the OF is sensed either ventrally or laterally, perpendicular to the heading direction. The field of view (FOV) of the eyes and the provocatively small number of pixels (2 pixels per eye) and EMDs (one EMD per eye) obviously need to be increased when dealing with navigation in more sparsely textured environments. Increasing the number of motion sensors was found to improve the goal-directed navigation performances of an airship in noisy environments [48], and we recently described how an additional forward-looking EMD might enable our $\mathrm{MH}$ to climb steeper rises by providing the autopilot OCTAVE with an anticipatory feedforward signal [77]. It will also be necessary to enlarge the FOV and control the heading direction (in the yaw direction) to enable the $\mathrm{HO}$ to successfully negotiate more challenging corridors including L-junctions and T-junctions. The more frontally oriented visual modules required for this purpose could be based on measuring the OF divergence [59,60,61], a procedure that flies seem to use when they land and trigger body saccades [97, 81, 93].

In the field of aeronautics, these systems could serve to improve navigation aids and automatic maneuvers. Steady measurement of the ventral OF could prevent deadly crashes by warning pilots that the current altitude is "too low for the current groundspeed" without requiring any altitude and speed measurements [31]. An OCTAVE OF regulator implemented onboard an aircraft would enable it to gradually take off "under automatic visual control", to veto any attempt to descend to a groundheight not commensurate with the current groundspeed, and to make it land safely [75, 31]. These systems could also potentially be harnessed to guiding MAVs indoors or through complex terrains such as mountain and urban canyons. Since these control systems are parsimonious and do not rely on GPS or bulky and power-hungry emissive sensors such as FLIRs, RADARs or LADARs, they meet the strict constraints imposed on the bird and insect scale in terms of their size, mass and consumption.

For the same reasons, these autopilots could potentially be adapted to micro-space vehicles (MSVs) performing rendezvous and docking missions in space, or exploration missions on other celestial bodies. A Martian lander equipped with a more elaborate OCTAVE autopilot could perform smooth automatic landing (see Section 3 and [75, 31]). A flying reconnaissance rover equipped with more elaborate OCTAVE and LORA III autopilots could take off autonomously and explore an area, skimming the ground and hugging the walls of a canyon, and adapting its groundspeed and clearance from the walls automatically to the width of the canyon (see Section 4). The orbiter (or base station) would simply have to send the rover a set of three low bandwidth signals: the values of the three OF set-points [26].

\section{Acknowledgments}

We are grateful to S. Viollet, F. Aubépart L. Kerhuel and G. Portelli for their fruitful comments and suggestions during this research. G. Masson participated in the experiments on bees and D. Dray in the experimental simulations on LORA III. We are also thankful to Marc Boyron (electronics engineer), Yannick Luparini and Fabien Paganucci (mechanical engineers) for their expert technical assistance and J. Blanc for revising the English manuscript. Serge Dini (beekeeper) gave plenty of useful advice during the behavioral experiments. This research was supported by CNRS (Life Science; Information and Engineering Science and Technology), an EU contract (IST/FET - 1999-29043) and a DGA contract $(2005-0451037)$. 


\section{References}

1. Aubépart, F., Franceschini., N.: Bio-inspired optic flow sensors based on FPGA: application to micro-air vehicles. J. Microprocessors and Microsystems 31, 408-419 (2007)

2. Aubépart, F., El Farji, M., Franceschini, N.: FPGA implementation of elementary motion detectors for the visual guidance of micro-air vehicles. In: Proc. IEEE Intern. Symp. Industrial Electronics (ISIE'2004), Ajaccio, France, 71-76 (2004)

3. Baird, E., Srinivasan, M.V., Zhang, S.W., Cowling., A.: Visual control of flight speed in honeybees. J. Exp. Biol. 208, 3895-3905 (2005).

4. Baird, E., Srinivasan, M.V., Zhang, S.W., Lamont R., Cowling. A.: Visual control of flight speed and height in the honeybee. In: From Animals to Animats 9, S. Nolfi et al. (SAB 2006), LNAI 4095, 40$51(2006)$

5. Barber, D.B.; Griffiths, S..R.; McLain, T.W.;\& Beard, R.W.: Autonomous landing of miniature aerial vehicles. In: Proc. American Institute of Aeronautics and Astronautics Conf. (2005)

6. Barrows, GL, Neely, C., Miller, K.T.: Optic flow sensors for MAV navigation. In: Fixed and flapping wing aerodynamics for micro-air vehicle applications. Progress in Astronautics and Aeronautics, Vol. 195, 557-574 (2001)

7. Blanès, C.: Appareil visuel élémentaire pour la navigation à vue d'un robot mobile autonome. M.S. thesis in Neuroscience, Université d'Aix-Marseille II, Marseille (1986)

8. Blanès, C.: Guidage visuel d'un robot mobile autonome d'inspitation bionique. PhD thesis, Polytechnical Institute, Grenoble (1991). Thesis work at the Neurocybernetics lab, CNRS, Marseille, France

9. Borst, A., Haag, J.: Neural networks in the cockpit of the fly. J. Comparative Physiology A188, 419437 (2002)

10. Buchner, E., Götz, K.G.: Evidence for one-way movement detection in the visual system of Drosophila. Biological Cybernetics 31, 243-248 (1978)

11. Collett, T., Nalbach, H.O., Wagner, H.: Visual stabilization in Arthropods ; In :Visual motion and its role in the stabilization of gaze, Miles, F.A. and Wallman, J. Elsevier, pp. 239-263 (1993)

12. Coombs, D., Roberts, K.: Centering behavior using peripheral vision. In : Proc. IEEE Conf. on Computer Vision and Pattern Recognition, New York, USA, (1993)

13. Dahmen H.J. et al.: this volume

14. David, C.: The relationship between body angle and flight speed in free-flying Drosophila. Physiological Entomology 3, 191-195 (1978)

15. David, C.: Height control by free-flying Drosophila, Physiological Entomology 4, 209-216 (1979)

16. David, C.: Compensation for height in the control of groundspeed by Drosophila in a new, 'barber's pole' wind tunnel. J. Comp. Physiol. A 147, 1432-1351 (1982).

17. David, C.: Visual control of the partition of flight force between lift and thrust in free-flying Drosophila. Nature 313, 48-50 (1985)

18. Dickson, W., Straw, A., Poelma, C., Dickinson, M.: An integrative model of insect flight control. In : Proc. $44^{\text {th }}$ Am. Inst. of Aeronautics, Astronautics and Aerospace Meeting, Reno, USA (2006)

19. Duchon, A.P., Warren, W.H.: Robot navigation from a Gibsonian viewpoint. In: Proc. IEEE Intern. Conf. on Systems, Man, and Cybernetics (SMC), San Antonio, USA, 2272-2277 (1994)

20. Egelhaaf, M., Borst, A.: Movement detection in Arthropods. In: Visual motion an its role in the stabilization of gaze (F.A. Miles and J. Wallman, Eds.) Amsterdam : Elsevier, 53-77 (1993)

21. Egelhaaf, M. et al.: This volume

22. Epstein, M., Waydo, S., Fuller, S.B., Dickson, W., Straw, A., Dickinson, M.H., Murray R.M.: Biologically inspired feedback design for Drosophila flight. In: Proc. Am. Control Conf. (ACC), NY, USA, 3395-3401 (2007).

23. Esch, H., Natchigall, W., Kogge, S.N.: Correlations between aerodynamic output, electrical activity in the indirect flight muscles and flight positions of bees flying in a servomechanically controlled flight tunnel. J. Comp. Physiol., 100, 147-159 (1975)

24. Franceschini, N.: Early processing of colour and motion in a mosaic visual system. Neuroscience Research, Suppl. 2, 517-549 (1985)

25. Franceschini, N.: Sequence-discriminating neural network in the eye of the fly, in: Analysis and Modeling of Neural Systems, F.H.K. Eeckman (Ed.), Norwell, USA : Kluwer Acad. Pub., pp.142150, (1992)

26. Franceschini, N.: Towards automatic visual guidance of aerospace vehicles: from insects to robots. Proceedings of the ACT Workshop on Innovative Concepts « A Bridge to Space » ESAESTEC, Noordwijk, The Netherlands, Acta Futura 2008, pp. 8-23 in press (2008) 
27. Franceschini, N., Blanes, C. and L. Oufar, L.: Appareil de mesure, passif et sans contact, de la vitesse d'un objet quelconque. Technical Report ANVAR/DVAR, N51549, Paris, France (1986)

28. Franceschini, N., Pichon, J.M., Blanès, C.: From insect vision to robot vision. Phil. Trans. Royal Soc. London B 337, 283-294 (1992)

29. Franceschini, N., Riehle, A., Le Nestour A.: Directionally selective motion detection by insect neurons. In: Facets of Vision, D.G. Stavenga and R.C. Hardie (Eds.), Berlin : Springer, Chapt. 17, pp.360-390 (1989)

30. Franceschini, N., Ruffier, F., Serres, J.: A bio-inspired flying robot sheds light on insect piloting abilities. Current Biology 17, 329-335 (2007)

31. Franceschini, N., Ruffier, F., Viollet, S., Boyron, M.: Steering aid system for altitude and horizontal speed, perpendicular to the vertical, of an aircraft and aircraft equipped therewith. International Patent PCT FR2003/002611 (2003)

32. von Frisch, K.: Tanzsprache und Orientierung der Bienen, Berlin : Springer (1965).

33. Garratt M.A., Chahl, J.S.: Vision-based terrain following for an unmanned rotorcraft. J. Field Robotics 25, 284-301 (2008)

34. Gibson, J.J.: The perception of the visual world, Boston: Houghton Mifflin (1950).

35. Gibson J.J., Olum, P., Rosenblatt, F.: Parallax and perspective during aircraft landings Amer. J. Psychol. 68, 372-395 (1955)

36. Götz, K.G.: Flight control in Drosophila by visual perception of motion. Kyb. 4, 199-208 (1968)

37. Green, WF, Oh, Y., Barrows, G.: Flying insect inspired vision for autonomous axial robot maneuvers in near earth environments. Proc. IEEE Int. Conf. Rob. Automation 2343-2352 (2004)

38. Griffiths, S., Saunders, J., Curtis, A., Barber, B., McLain, T., Beard, R.: Maximizing miniature aerial vehicles - obstacle and terrain avoidance for MAVs. IEEE Robotics and Automation Magazine 13, 34-43 (2006)

39. Hausen K., Egelhaaf, M.: Neural mechanisms of visual course control in insects. In: Facets of Vision, D.G. Stavenga and R.C. Hardie (Eds), Berlin: Springer, chapt. 18, pp. 391-424 (1989)

40. Hassenstein, B., Reichardt, W.: Systemtheoretische Analyse der Zeitreihenfolgen und Vorzeichen-Auswertung bei der Bewegungsperzeption des Rüsselkäfers Chlorophanus. Z. für Naturforschung 11b, 513-524 (1956)

41. Heisenberg, M. and Wolf, R.: Vision in Drosophila. Genetics of microbehavior. Berlin : Springer (1984)

42. Hengstenberg R.: Mechanosensory control of compensatory head roll during flight in the blowfly Calliphora erythrocephala Meig. J. Comp. Physiol. A 163, 151-165 (1988).

43. Hengstenberg R.: Control of head pitch in Drosophila during rest and flight. Göttingen Neurobiology Meeting 305 (1992)

44. Heran, P., Lindauer, M.: Windkompensation und Seitenwindkorrektur der Bienen beim Flug über Wasser. Z. für vergl. Physiol. 47, 39-55 (1963)

45. Humbert, J.S., Hyslop, H., Chinn, M.: Experimental validation of wide-field integration methods for autonomous navigation. In : Proc. IEEE/RSJ Intern. Conf. Intelligent Robots and Systems (IROS) San Diego, USA, 2144-2149 (2007)

46. Humbert, J.S., Murray, R.M., Dickinson, M.H.: Sensorimotor convergence in visual navigation and flight control systems. In : Proc. $16^{\text {th }}$ IFAC World Congress, Prague, Czech Republic (2005)

47. Ibbotson, M.R.: Evidence for velocity-tuned motion-sensitive descending neurons in the honeybee. Proc.Roy. Soc., London B 268, 2195-2201 (2001)

48. lida, F.: Goal-directed navigation of an autonomous flying robot using biologically inspired cheap vision. Proc. 32nd International Symposium on Robotics (2001)

49. Kramer, J., Sarpeshkar, R., Koch, C.: Pulse-based analog VLSI velocity sensors. IEEE Trans. Circuits and Systems II : Analog and digital signal processing 44, 86-101 (1997)

50. Kellog, F.E, Fritzel, D.E., Wright R.H.: The olfactory guidance of flying insects IV. Drosophila. Canadian Entomology, 884-888 (1962)

51. Kennedy, J.S.: Visual responses of flying mosquitoes : In : Proc. Zool. Soc. of London 109, 221-242 (1939)

52. Kennedy, J.S.: The migration of the desert locust (Schistocerca gregaria Forsk.) I. The behaviour of swarms, Phil.Trans. Roy. Soc., London, B 235, 163-290 (1951)

53. Kennedy, J.S., Marsh D.: Pheromone-regulated anemotaxis in flying moths. Science 184, 9991001 (1974)

54. Kerhuel, L., Viollet, S., Franceschini, N.: A sighted aerial robot with fast gaze and heading stablization. In : Proc. IEEE Int. Conf. on Intelligent Robots Systems, San Diego, pp. 2634-2641 (2007) 
55. Kirchner, W.H. ; Srinivasan, M.V.: Freely moving honeybees use image motion to estimate distance. Naturwissenchaften 76, 281-282 (1989)

56. Koenderink, J.J : Optic flow. Vision Research 26, 161-179 (1986)

57. Moeckel, R. , Liu S.C. (this volume)

58. Mura, F., Franceschini., N.: Visual control of altitude and speed in a flying agent. In : From

Animals to Animats III, D. Cliff et al. (Eds), Cambridge: MIT Press, 91-99 (1994)

59. Nelson, RC, Aloimonos, Y. Obstacle avoidance using flow field divergence. IEEE Transactions on Pattern Analysis and Machine Intelligence 11, 1102-1106 (1989)

60. Netter, T., Franceschini, N.: Neuromorphic optical flow sensing for Nap-of-the-Earth flight. In :

Mobile Robots XIV, D.W. Gage and H.M. Choset (Eds), SPIE, Vol. 3838, 208-216 (1999)

61. Netter, T., Franceschini, N.: A robotic aircraft that follows terrain using a neuromorphic eye. In

Proc. IEEE Intern. Conf. Intelligent Robots \& Systems (IROS) Lausanne, Switzerland, 129-134 (2002)

62. Neumann T.R., Bülthoff, H.: Insect inspired visual control of translatory flight, in : Proc. ECAL

2001, Berlin : Springer, pp. 627-636 (2001)

63. Pichon, J-M., Blanès, C., Franceschini, N.: Visual guidance of a mobile robot equipped with a network of self-motion sensors. In : Mobile Robots IV, W.J. Wolfe, W.H. Chun (Eds.) Bellingham, U.S.A : SPIE Vol. 1195, 44-53 (1989)

64. Portelli, G., Serres, J., Ruffier F., Franceschini, N.: An Insect-Inspired Visual Autopilot for Corridor-Following. Proc. IEEE Intern. Conf. Biomedical Robotics \& Biomechatronics, BioRob 08, Scottsdale, USA, pp. 19-26 (2008)

65. Preiss, R.: Set-point of retinal velocity of ground images in the control of swarming flight of desert locusts. Journal Comparative Physiology A 171, 251-256 (1992).

66. Preiss, R., Kramer, E.: Control of flight speed by minimization of the apparent ground pattern movement. In Localization and orientation in Biology and Engineering, D. Varju and H. Schnitzler, eds. Berlin : Springer, pp. 140-142 (1984)

67. Pudas, M, Viollet, S, Ruffier, F, Kruusing, A, Amic, S, Leppävuori, S, Franceschini, N.: A miniature bio-inspired optic flow sensor based on low temperature co-fired ceramics (LTCC) technology, Sensors and Actuators A133, 88-95 (2007)

68. Reichardt, W.: Movement perception in insects. In: Processing of Optical Data by Organisms and by Machines, W. Reichardt (Ed.), New York: Academic Press (1969)

69. Reiser, M, Humbert, S., Dunlop, M., Vecchio, D., Murray, M., Dickinson, M.: Vision as a compensatory mechanism for disturbance rejection in upwind flight. American Control Conference 04. Proc. IEEE Comp. Soc., 311-316 (2004)

70. Riehle, A, Franceschini, N.: Motion detection in flies: parametric control over ON-OFF pathways. Exp. Brain Res. 54, 390-394 (1984)

71. Riley, J.R., Osborne, JL.: Flight trajectories of foraging insects: observations using harmonic radar. In: Insect Movement: Mechanisms and Consequences, D.R. Reynolds and C.D. Thomas (Eds.), CAB International, Chapter 7, 129-157 (2001).

72. Rossel, S., Wehner, R. : How bees analyze the polarization pattern in the sky. Experiments and model. J. Comp. Physiol. A154, 607-615 (1984)

73. Ruffier, F., Franceschini, N.: OCTAVE, a bioinspired visuo-motor control system for the guidance of Micro-Air Vehicles, In: Bioengineered and Bioinspired Systems, A. Rodriguez-Vazquez, D. Abbott, R. Carmona, (Eds.), Bellingham, U.S.A : SPIE, Vol. 5119, pp. 1-12 (2003)

74. Ruffier, F., Franceschini, N.: Visually guided micro-aerial vehicle: automatic take-off, terrain following, landing and wind reaction. In: Proc. IEEE Internat. Conf. Robotics \& Automation (ICRA), New Orleans, USA, pp. 2339-2346 (2004)

75. Ruffier, F. \& Franceschini, N.: Automatic landing and takeoff at constant slope without terrestrial aids. In: Proc. 31th Eur. Rotorcraft Forum, AAF/CEAS, Florence, pp. 92.1-92.8. (2005)

76. Ruffier, F., Franceschini, N.: Optic flow regulation: the key to aircraft automatic guidance.

Robotics and Automomous Systems 50, 177-194 (2005)

77. Ruffier, F., Franceschini, N.: Aerial robot piloted in steep relief by optic flow sensors. In: Proc. IEEE Intern. Conf. Intelligent Robots and Systems (IROS), Nice, pp.1266-1273 (2008)

78. Ruffier, F., Viollet, S., Amic, S., Franceschini, N.: Bio-inspired optical flow circuits for the visual guidance of micro-air vehicles. Proc. IEEE, ISCAS, Bangkok, Thaïland, III, pp. 846-849 (2003) 79. Santos-Victor, J., Sandini, G., Curotto, F., Garibaldi, S.: Divergent stereo in autonomous navigation: from bees to robots. International J. of Computer Vision 14, 159-177 (1995)

80. Sarpeshkar, R. ; Kramer, J. ; Koch, C.: Pulse domain Neuromorphic Circuit for Computing Motion, US patent Nb 5,781,648 (1998) 
81. Schilstra, C., van Hateren J.H.: Blowfly flight and optic flow. I. Thorax kinematics and flight dynamics. J. Exp. Biol. 202, 1481-1490 (1999)

82. Serres, J., Dray, D, Ruffier, F., Franceschini, N.: A vision-based autopilot for a miniature airvehicle: joint speed control and lateral obstacle avoidance. Autonomous Robots 25, 103-122 (2008) 83. Serres, J., Masson, G., Ruffier, F., Franceschini, N.: A bee in the corridor : centering and wall following. Naturwissenschaften, 95 :1181-118 (2008)

84. Spork, P., Preiss, R.: Control of flight by means of lateral visual stimuli in gregarous desert locusts, Schistocerca gregaria. Physiological Entomology 18, 195-203 (1993)

85. Srinivasan, M.V.: How insects infer range from visual motion. In: Visual motion and its role in the stabilization of gaze. F.A. Miles and J. Wallman, Eds. Amsterdam : Elsevier (1993)

86. Srinivasan, M.V. et al.: This volume

87. Srinivasan, M.V., Lehrer, M., Kirchner, W.H., Zhang, S.W.: Range perception through apparent image speed in freely flying honeybees. Visual Neuroscience 6, 519-535 (1991)

88. Srinivasan, M.V., Zhang, S.W., Lehrer, M., Collett., T.: Honeybee navigation en route to the goal: visual flight control and odometry. J. Exp. Physiol. 199, 237-244 (1996)

89. Srinivasan, M.V., Chahl, J.S., Weber, K., Venkatesh, S., Nagle, M.G., Zhang, S.W.: Robot navigation inspired by principles of insect vision. Robot. \& Autonom. Syst., 26: 203-216 (1999)

90. Srinivasan, M.V., Zhang, S.W., Chahl, J.S., Barth, E., Venkatesh, S.: How honeybees make grazing landings on flat surface. Biological Cybernetics 83, 171-183 (2000)

91. Strausfeld, N.: Beneath the compound eye: Neuroanatomical analysis and physiological correlates in the study of insect vision. In: Facets of Vision, D.G. Stavenga and R.C. Hardie (Eds), Berlin: Springer, chapt. 16, pp. 316-359 (1989)

92. Tammero, L.F., Dickinson, M.H.: Collision-avoidance and landing responses are mediated by separate pathways in the fruit fly, Drosophila melanogaster. J. Exp. Biol. 205, 2785-2798 (2002)

93. Tammero, L.F., Dickinson, M.H.: The influence of visual landscape on the free flight behavior on the fruit fly Drosophila melanogaster. J. Exp. Biol. 205, 327-343 (2002)

94. Taylor G.K., Krapp, H.G.: Sensory systems and flight stability: what do insects measure and why ? In : Advances in Insect Physiol. 34 : Insects mechanisms and control, J. Casas and S.J. Simpson (Eds.) Amsterdam : Elsevier, 231-316 (2008)

95. Ullman, S.: Analysis of visual motion by biological and computer systems, Computer 14, 57-69 (1981).

96. van Hateren, H., Schilstra, C.: Blowfly flight and optic flow, II. Head movement during flight. J. Exp. Biol. 202: 1491-1500 (1999)

97. Wagner, H.: Flow-field variables trigger landing in flies. Nature 297, 147-148 (1982)

98. Wagner, H.: Flight performance and visual control of flight of the free-flying housefly Musca domestica, I. Organisation of the flight motor. Phil.Trans Roy. Soc. B312, 527-551 (1986)

99. Whiteside, T.C. and G.D. Samuel.: Blur zone. Nature 225, 94-95, (1970)

100. Zeil, J., Boeddeker, N., Hemmi, J.M.: Vision and the organization of behavior, Current Biology 18, 320-323 (2008)

101. Zettler, F., Weiler, R.: Neuronal processing in the first optic neuropile of the compound eye of the fly. In: Neural principles in vision, F. Zettler and R. Weiler Eds., Berlin, Springer, 226-237 (1974)

102. Zufferey, J.C. et al.: this volume 


\section{Figure legends}

Figure 1. (left): Experimental scheme used to analyze the principles underlying elementary motion detectors (EMDs) in flies, using single neuron recording and single photoreceptor stimulation (d) The triple-beam incident light "microscope-telescope" used to deliver a sequence of $1 \mu \mathrm{m}$ light spots to two neighboring photoreceptors (the fly's head is indicated by the white arrow). The microelectrode (c) recorded the electrical response (nerve impulses) of the motion sensitive neuron $\mathrm{H} 1$ to this "apparent motion" (From [29]).

Figure 2 : Principle $(a, b)$ of the elementary motion detector (EMD) inspired by our electrophysiological findings (cf figure 1) (after [7, 27, 8]) and (d) the purely analog version made in the 1989's with small mounted device (SMD) technology (From [8]). (c, e) hybrid version (mass 0.8 grams) based on a microcontroller (From [78]). (f) similar hybrid version (size: $7 \mathrm{~mm} \times 7 \mathrm{~mm}$, mass 0.2 grams) built using Low Temperature Co-fired Ceramics technology (LTCC) (From [67]).

Figure 3. (a) The ventral OF is an angular speed $\omega\left[\right.$ rad. $\mathrm{s}^{-1}$ ] corresponding to the groundspeed-togroundheight ratio. (b) The OF sensor comprises a microlens and two photoreceptors separated by a small angle $\Delta \varphi$ (see figure 2a) driving an EMD. The latter delivers a signal $\omega_{\text {meas }} \cong \Delta \varphi / \Delta t=V_{x} / \boldsymbol{h}$. which serves as a feedback signal in the OF regulator (figure 4a). The one-dimensional random texture is a magnified sample of that shown in figures $5 b \& 6 a$ (From [30]).

Figure 4: (a) The OCTAVE autopilot consists of a feedback control system, called the optic flow regulator (bottom part) that controls the vertical lift, and hence the groundheight, so as to maintain the ventral OF, $\omega$, constant and equal to the set-point $\omega_{\text {set }}$ whatever the groundspeed. (b) Like flies [14] and bees [23], our micro-helicopter $(\mathrm{MH})$ gains speed by pitching its mean flight force vector forward at an angle $\Theta$ with respect to the vertical. Controlling $\boldsymbol{F}$ (via the rotor $r p m$ ) amounts to mainly controlling $L$ because $\Theta$ remains smal $\left(\Theta_{\max }<10^{\circ}\right.$ for $\left.V_{x} \max =3 \mathrm{~m} / \mathrm{s}\right)$ (From [30]).

Figure 5 : (a) 100-gram Micro-helicopter (MH) equipped with a ventral OF sensor (figure 2e) and an OF regulator (figure 4a). The 5-gram rotor is driven by a DC motor via a reducer. (b) The MH, which is mounted on the light pantographic arm of a flight mill, can be remotely pitched forward at angle $\Theta$ while keeping its roll attitude. The $\mathrm{MH}$ circles over a large arena (outside diameter: $4.5 \mathrm{~m}$ ). In the experiments presented here (figure 6), the arena was flat, without any rising slopes. (From [74]).

Figure 6: Flight path (a) and flight parameters monitored during a 70-meter flight (consisting of about 6 laps over the test arena: figure 5b) performed by the micro-helicopter (MH) equipped with the $O F$ regulator (figure 4a). The complete flight-path (a) over the randomly textured pattern includes take-off, level flight and landing. (b) Groundspeed $\boldsymbol{V}_{\boldsymbol{x}}$. (c) Output $\boldsymbol{\omega}_{\text {meas }}$ of the OF sensor. (d) Actual OF output $\omega$ (calculated from $\boldsymbol{V}_{x} / \boldsymbol{h}$ ) generated by the OF regulator (From [30]).

Figure 7: Honeybees' centering and wall-following behavior. Bees were trained to enter an apparatus where sugar solution was provided at the end of a wide (width $0.95 \mathrm{~m}$ ) 3-meter long corridor formed by two $0.25-\mathrm{m}$ high walls. A digital camera placed above the insect netting covering the corridor filmed the trajectory of individual flying bees over the central part of the corridor. The walls were lined with regularly spaced vertical white-and-grey stripes (period $0.1 \mathrm{~m}$; contrast $m=0.27$ ). The bee's entrance $\left(E_{C}\right)$ and the feeder $\left(F_{C}\right)$ were placed either on the corridor midline $(a)$ or on one side $(b, c, d)$. In (d), part of the left wall was removed during the trials. The mean ordinate of the trajectories was distributed as shown above ( $\boldsymbol{n}=$ number of trajectories recorded under each of the experimental conditions) (From [83]).

Figure 8: (a) Fully actuated hovercraft $(\mathrm{HO})$ developed to test the LORA III autopilot. The HO was equipped with two elementary eyes looking at an angle of $+90 /-90^{\circ}$ to the side. Each of them comprised only 2 pixels driving a single OF sensor (cf. figure 2c,e). (b) The miniature HO $(36 \times 21 \times 14 \mathrm{~cm})$ moved along a corridor, the walls of which had a random texture. The vehicle's heading was maintained along the $\mathrm{X}$-axis via a heading lock system compensating for any yaw disturbances by activating the two rear thrusters differentially. The two lateral eyes were therefore oriented perpendicularly to the corridor axis. The $O F$ experienced by each eye is proportional to the groundspeed and inversely proportional to the distance from the walls (Eqs. 4,5) (From [82]). 
Figure 9: The LORA III autopilot is a dual OF regulator consisting of two interdependent visual feedback loops: the forward control loop and the side control loop, each of which controls one degree of freedom ( $x$ or $y$ ). The forward controller adjusts the forward thrust, and hence the forward speed $\boldsymbol{V}_{\boldsymbol{x}}$, so as to minimize the error signal $\boldsymbol{\varepsilon}_{F w d}$. The side controller adjusts the lateral thrust, and hence the side speed, and hence the ordinate $\boldsymbol{y}$, so as to minimize the error signal $\boldsymbol{\varepsilon}_{\text {side. }}$. The sign of the difference between the left and right OFs measured determines which wall will be followed. The robot's initial ordinate $y_{0}$, and the right and left wall ordinates $\left(y_{R}, y_{L}\right)$ are regarded by LORA III as disturbances impinging on the system at particular points (black arrows) (From [82]).

Figure 10: (a) Wall-following behavior of the hovercraft $(\mathrm{HO})$ (time marks on the flight paths are at 0.3second intervals). The $\mathrm{HO}$ moves to the right at a forward OF set-point $\omega_{\text {SetFwd }}=300 \%$ (3.28 Volts) and a sideways OF set-point $\omega_{\text {Setside }}=230 \%\left(2.21 \mathrm{~V}\right.$ ), starting at various ordinates $y_{0}$ (squares: $y_{0}=0.90 \mathrm{~m}$, crosses: $y_{0}=0.50 \mathrm{~m}$, full dots: $\left.y_{0}=0.10 \mathrm{~m}\right)$. Irrespective of its initial ordinate, the $\mathrm{HO}$ ends up following one wall with a clearance of $0.25 \mathrm{~m}$, at a forward speed $V_{x^{\infty}}=1 \mathrm{~m} / \mathrm{s}$. (c,d) The sum of the lateral OFs measured and the sum of the actual OFs both eventually equal the forward OF set-point $\omega_{\text {SetFwd }}=300 \%$ (e, f). The larger value of the OFs measured and that of the actual OFs both eventually equal the sideways OF set-point $\omega_{\text {SetFwd }}=230 \%$ s (From [82]).

Figure 11: "Centering behavior" as a particular case of "wall-following behavior" (a, b) Simulated trajectories of the hovercraft $(\mathrm{HO})$ moving to the right along a straight corridor at a forward OF setpoint $\omega_{\text {SetFwd }}=300 \%$, with various sideways OF set-points (crosses: $\omega_{\text {SetSide }}=130 \%$, open dots: $\omega_{\text {Setside }}=110 \% \mathrm{~s}$ ), full dots: $\omega_{\text {Setside }}=90 \% \mathrm{~s}$ ). Initial condition $y_{0}=0.25 \mathrm{~m}$, time marks as in figure 10 . The $\mathrm{HO}$ can be seen to consistently end up centering between the two walls at a forward speed of $1.3 \mathrm{~m} / \mathrm{s}$. (c,d) The larger of the two OFs measured and the larger actual OF both equal $150 \% \mathrm{~s}\left(=\omega_{\text {setFwd }} / 2\right)$. In attempting to reach the appropriate sideways OF set-point, the LORA III autopilot triggers changes in the sign of the error signal $\varepsilon_{\text {Side }}(E q .6)$, causing oscillations about the midline (see (a)) (From [82]).

Figure 12: Automatic navigation along a tapered corridor, requiring no data on the corridor width or the tapering angle $\boldsymbol{\alpha}$ (marks on trajectories as in figure 10). Again, the hovercraft's behavior is entirely determined by its two OF set-points: $\omega_{\text {SetFwd }}=300 \%$ and $\omega_{\text {SetSide }}=230 \%$. (a) Simulated trajectories of the $\mathrm{HO}$ moving to the right along the corridor (tapering angle $\alpha=7^{\circ}$ ) with three initial ordinates $y_{0}$ (open dots: $y_{0}=0.90 \mathrm{~m}$, crosses: $y_{0}=0.60 \mathrm{~m}$, full dots: $y_{0}=0.30 \mathrm{~m}$ ). (b) The forward speed decreases and increases linearly with the local corridor width and the distance $\boldsymbol{x}$ traveled. (c,d) The sum of the two lateral OFs measured (and that of the actual OFs computed with Eq. 4,5) are maintained constant and equal to $300 \%$ s $\left(=\omega_{\text {SetFwd }}\right)$. $(\mathbf{e}, \mathbf{f})$ The side control system effectively keeps whichever lateral OF is larger at a constant value of approximately $230 \%$ s $\left(=\omega_{\text {SetSide }}\right)$ (From [82]). 
FRANCESCHINI et al. (FIR FINAL, V2)

Figures 1-12
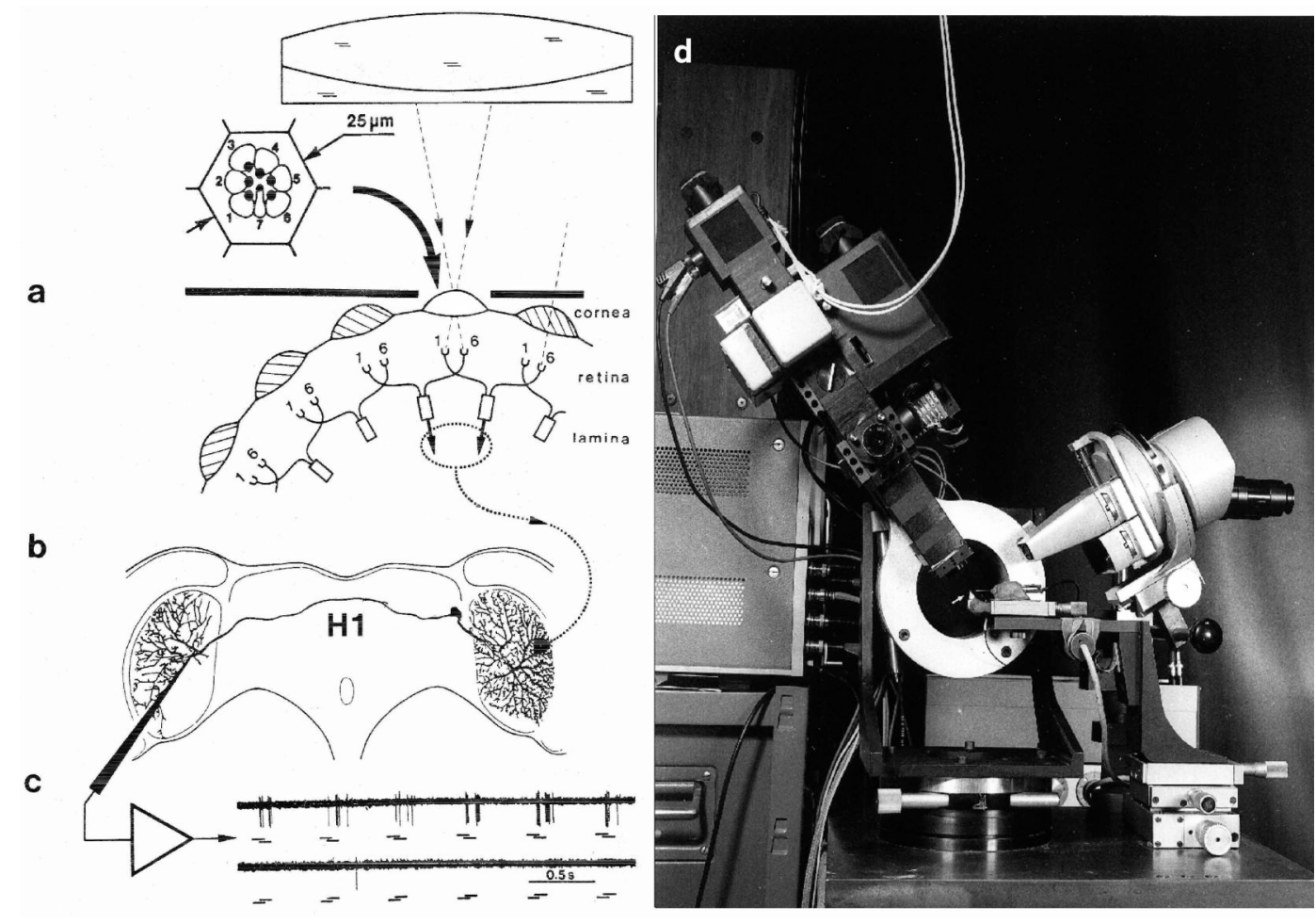

Fig. 1 
FRANCESCHINI et al. (FIR FINAL, V2)
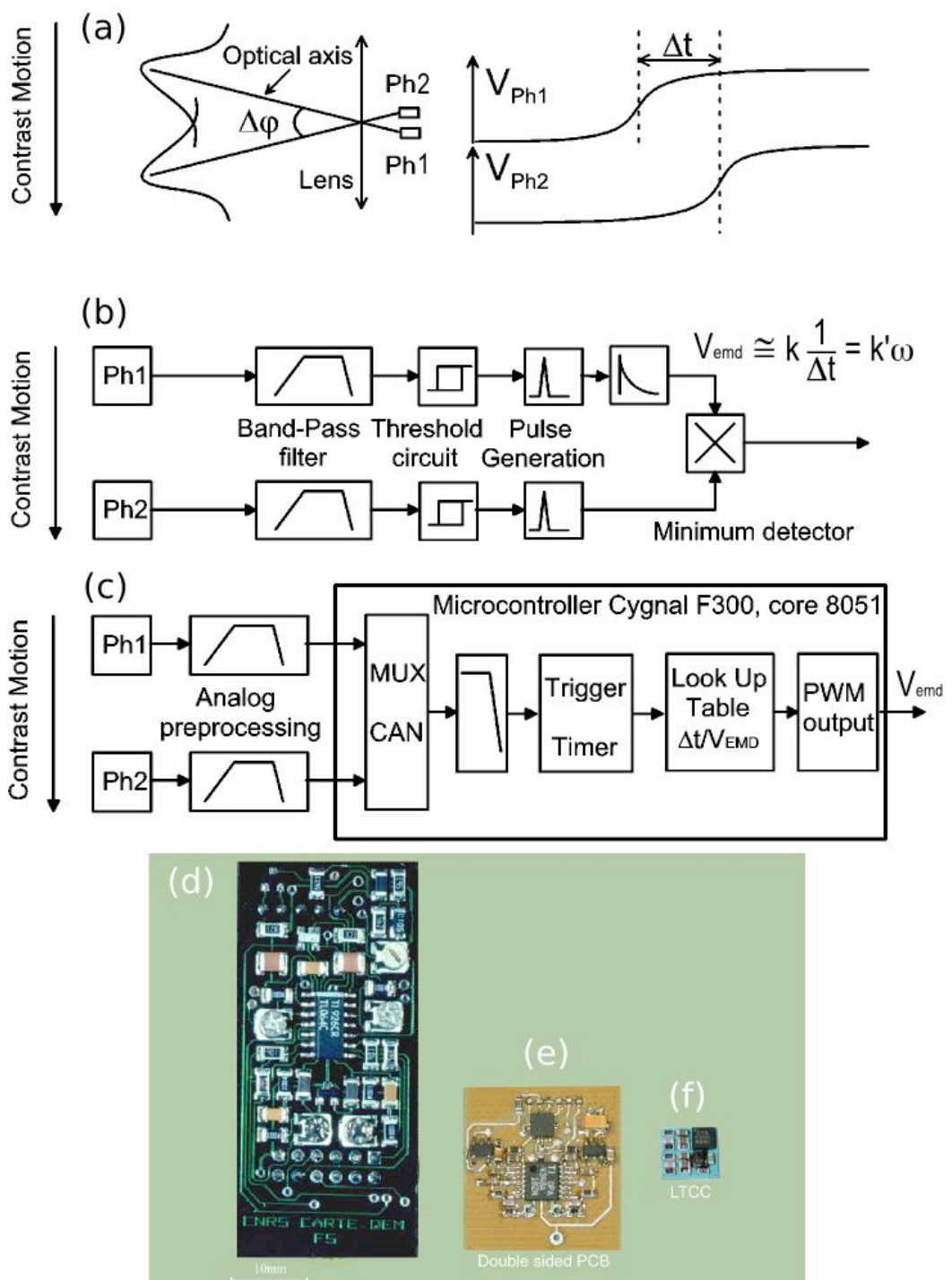

Fig. 2 
FRANCESCHINI et al. (FIR FINAL, V2)
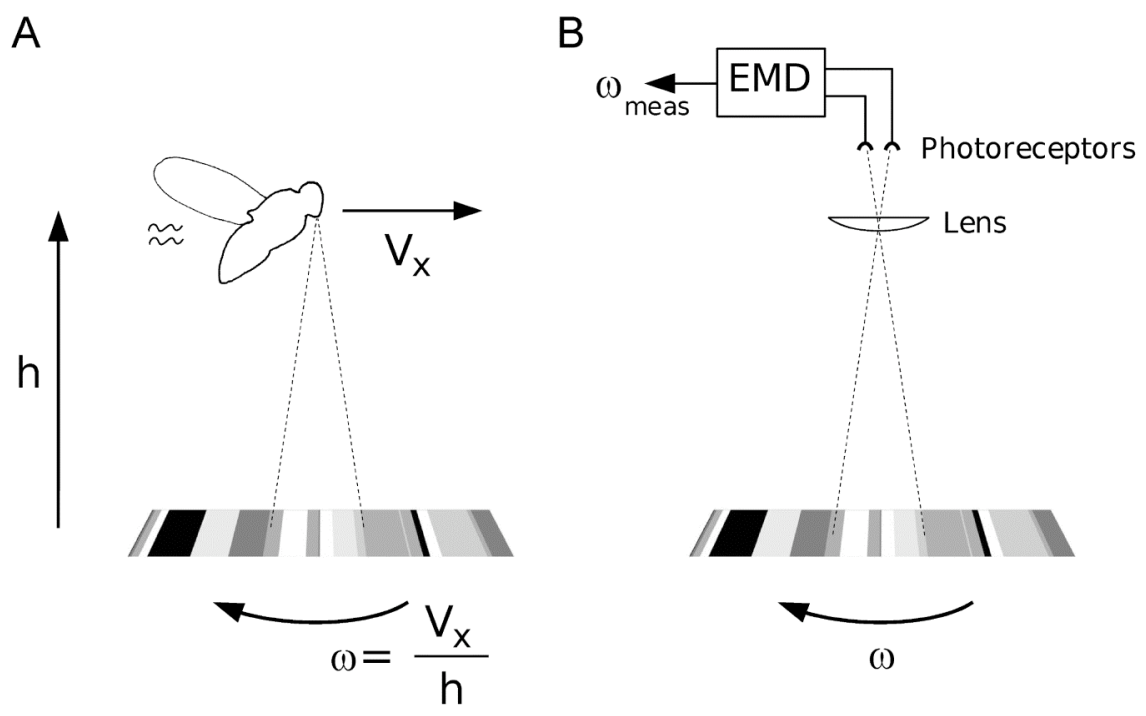

Fig. 3 
FRANCESCHINI et al. (FIR FINAL, V2)

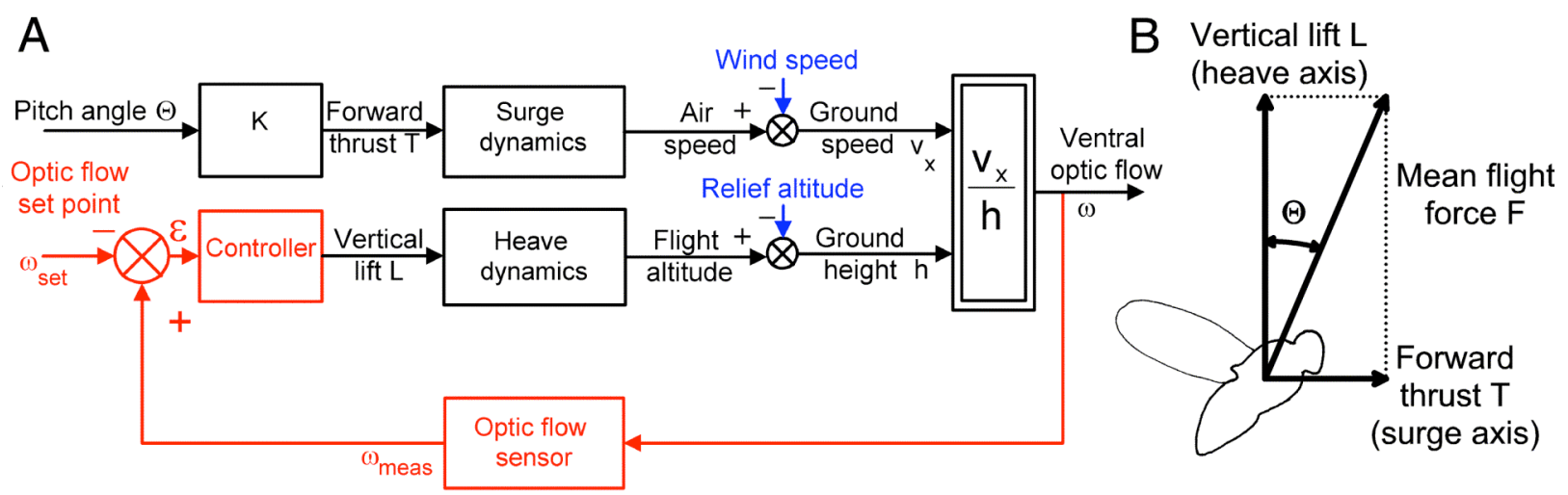

Fig. 4 
FRANCESCHINI et al. (FIR FINAL, V2)
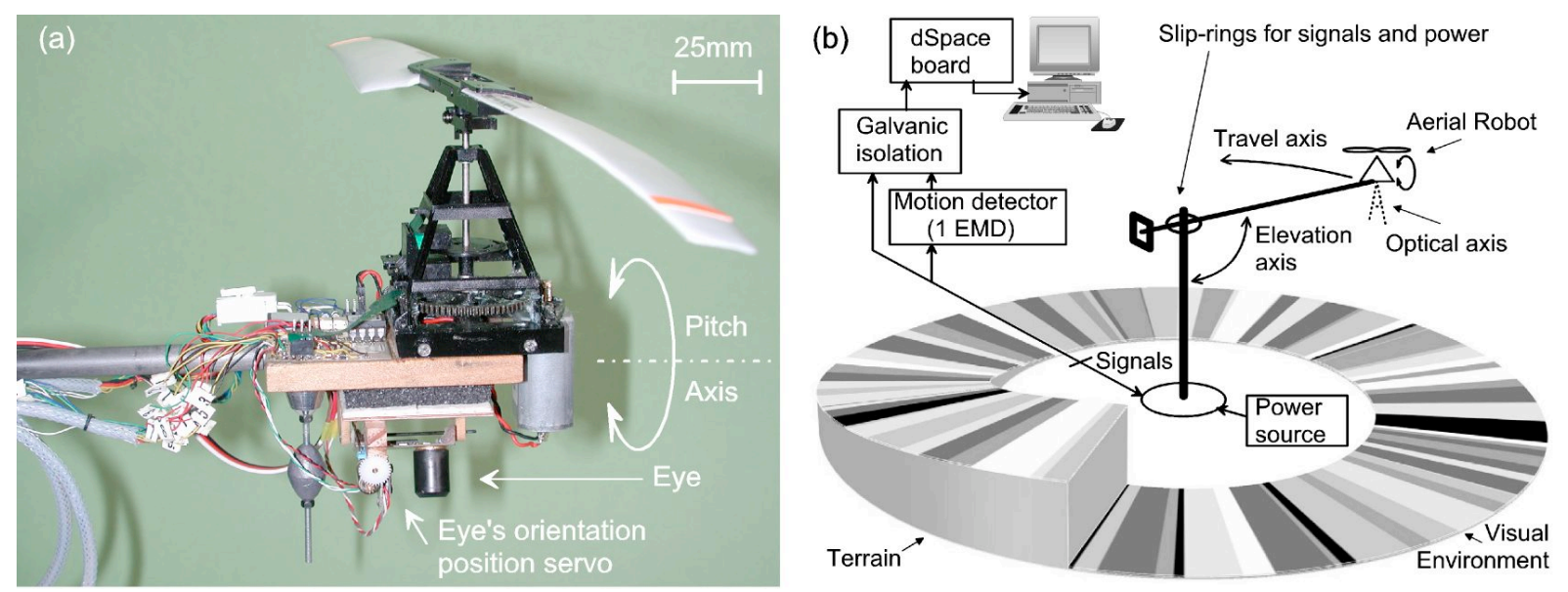

Fig. 5 
FRANCESCHINI et al. (FIR FINAL, V2)
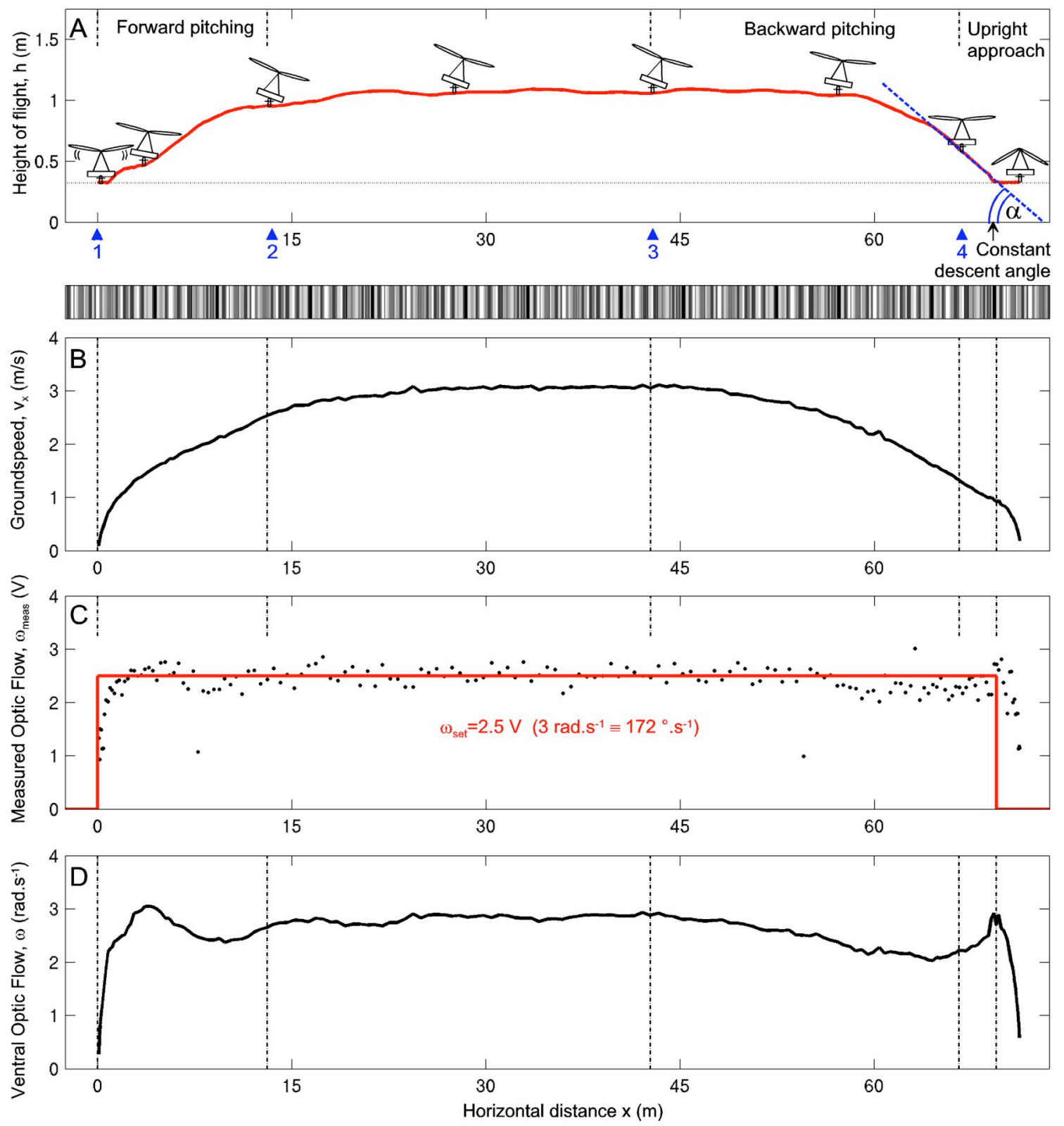

Fig. 6 
FRANCESCHINI et al. (FIR FINAL, V2)

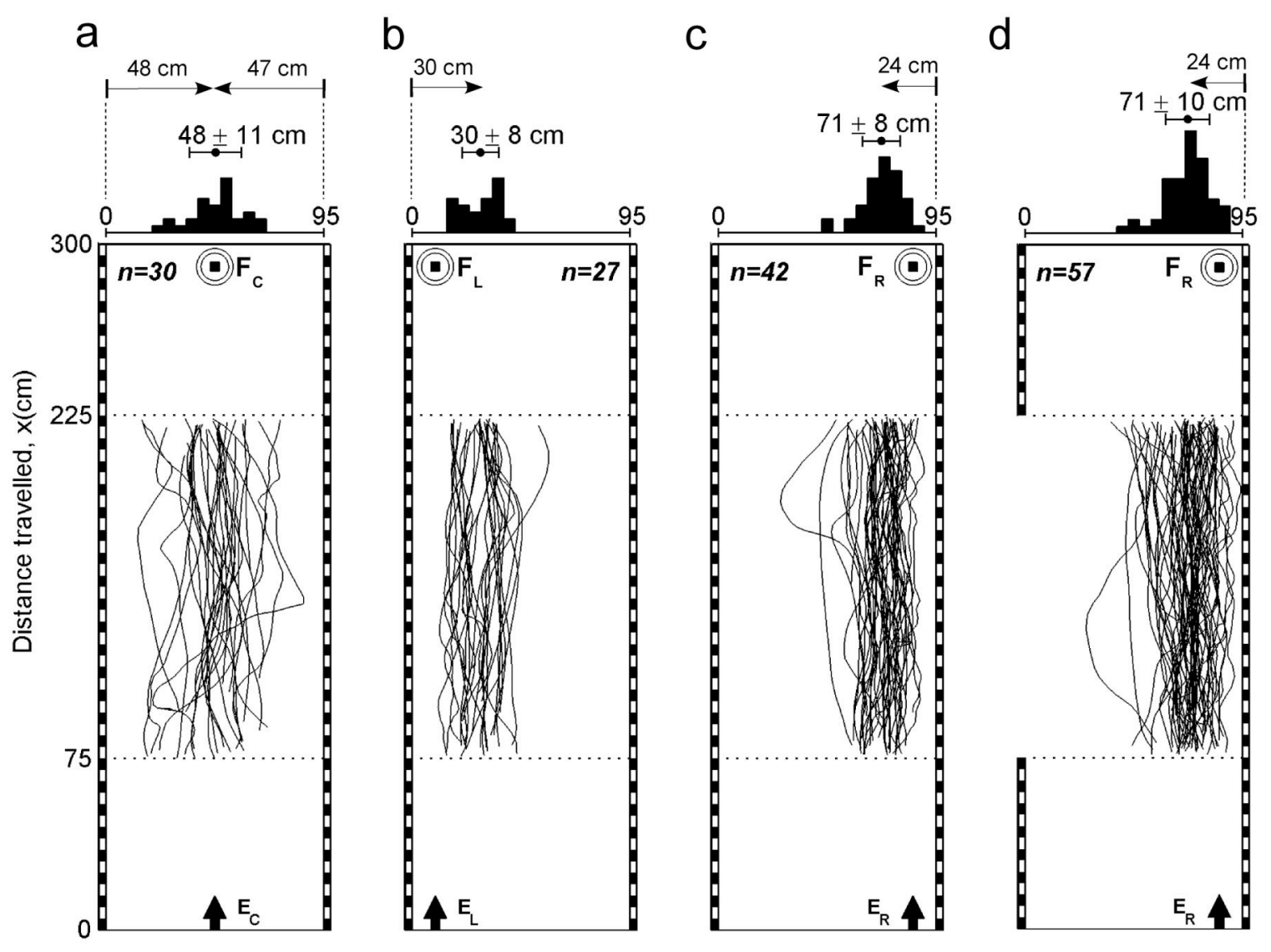

Fig. 7 
FRANCESCHINI et al. (FIR FINAL, V2)

Figures 8-12
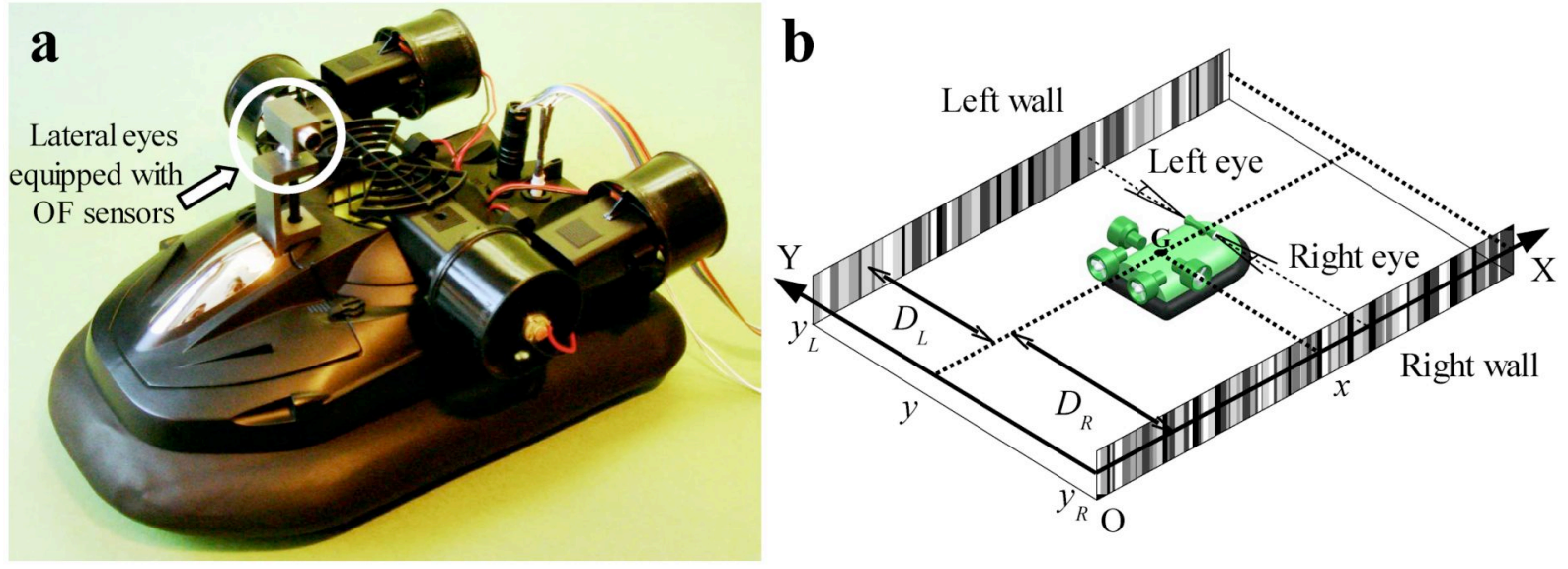

Fig. 8 
FRANCESCHINI et al. (FIR FINAL, V2)

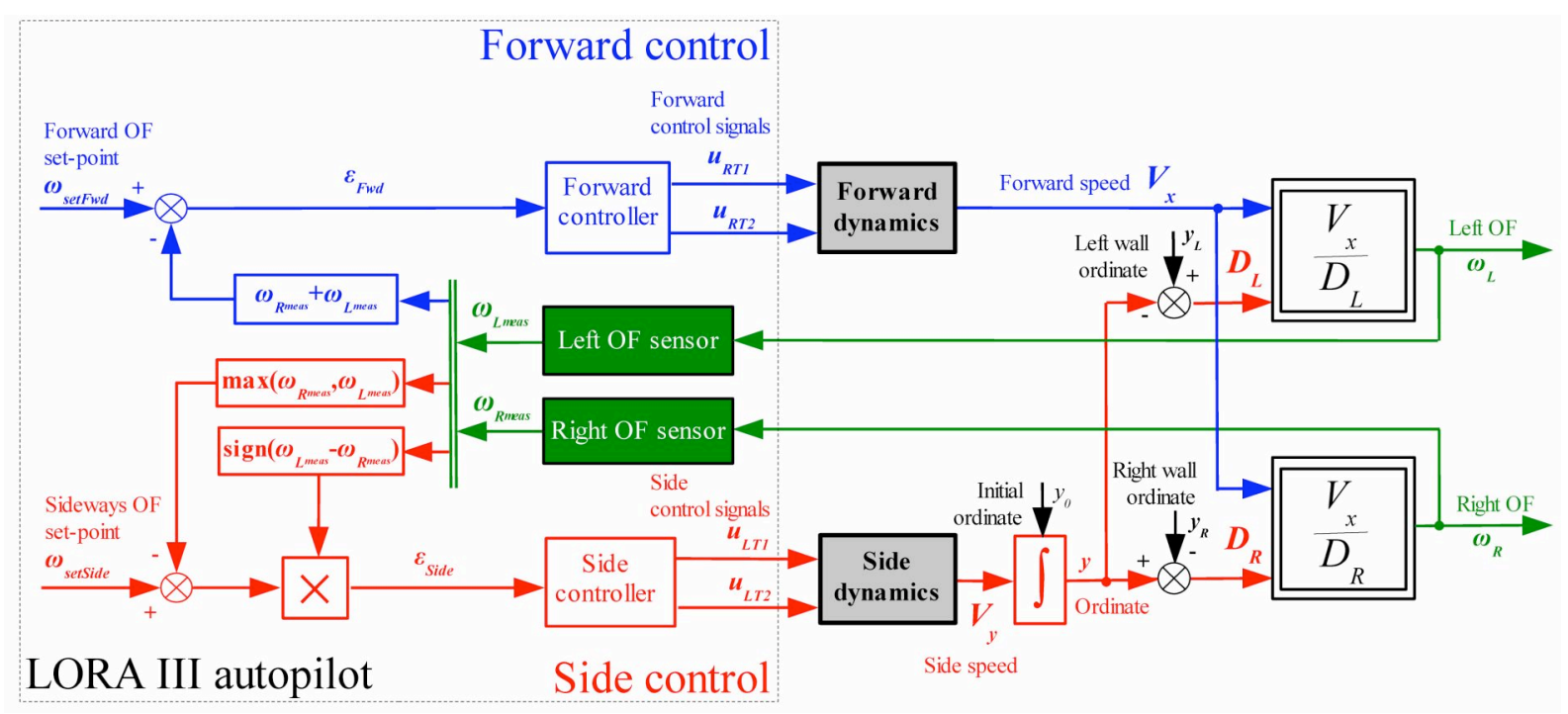

Fig. 9 
FRANCESCHINI et al. (FIR FINAL, V2)
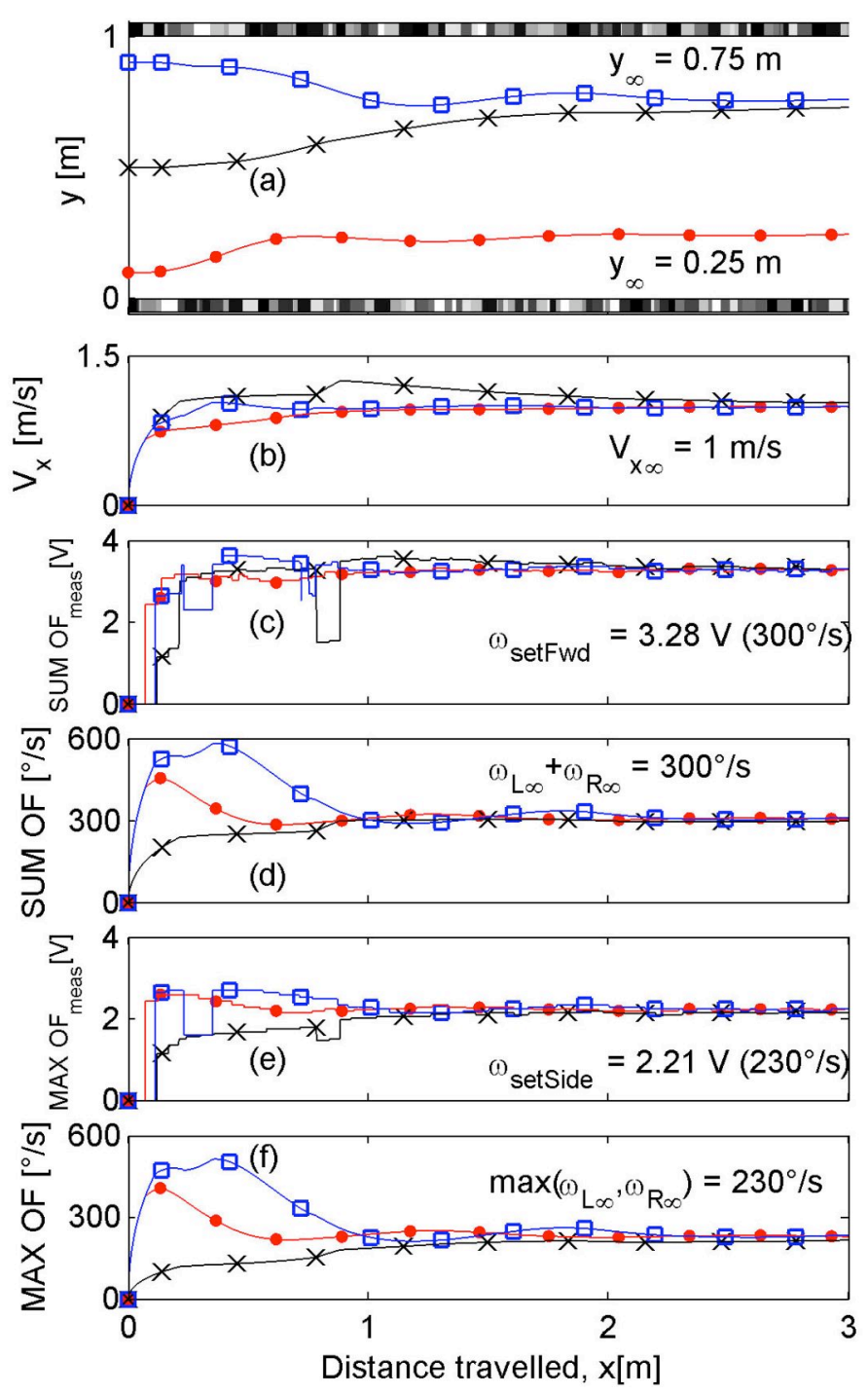

Fig. 10 
FRANCESCHINI et al. (FIR FINAL, V2)
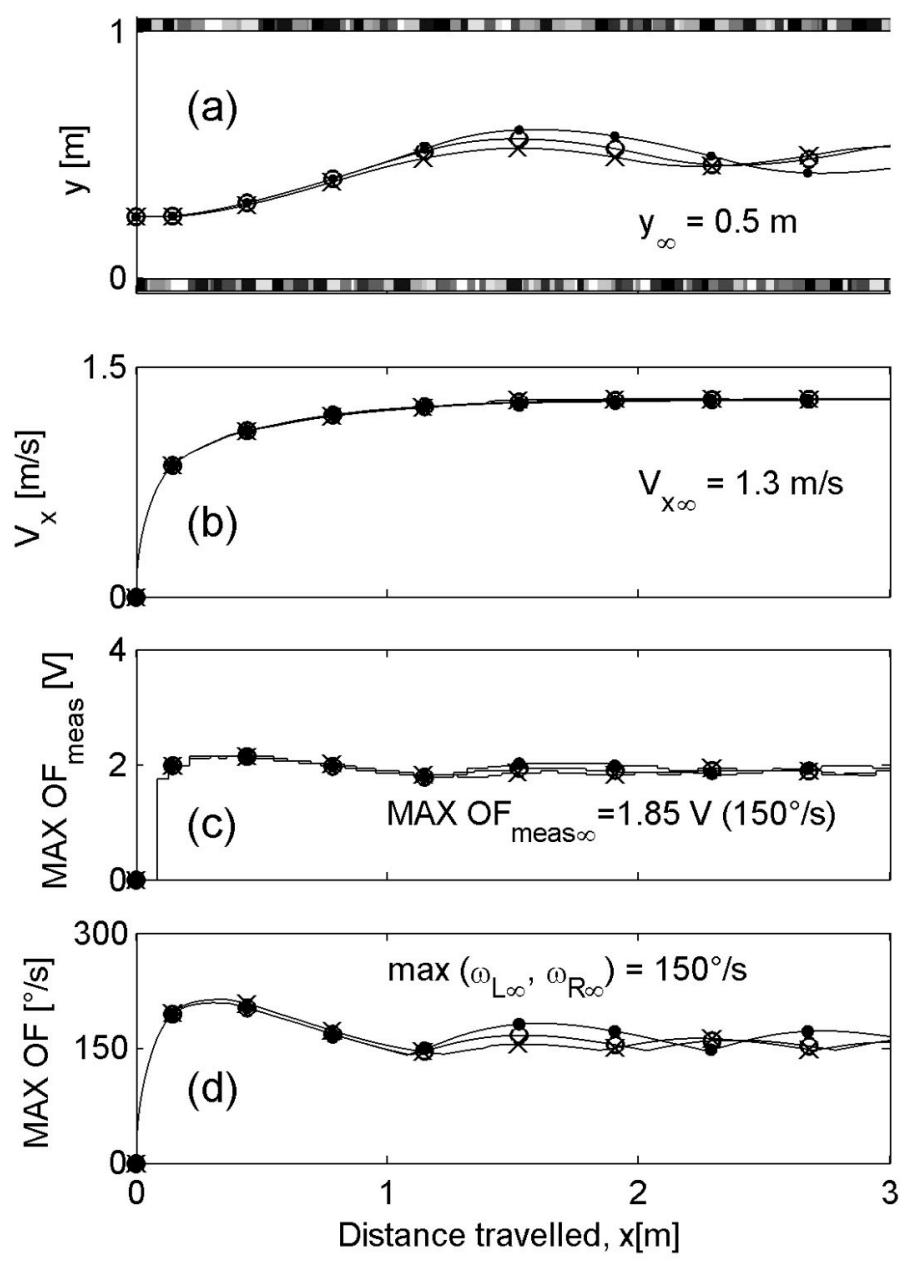

Fig. 11 
FRANCESCHINI et al. (FIR FINAL, V2)
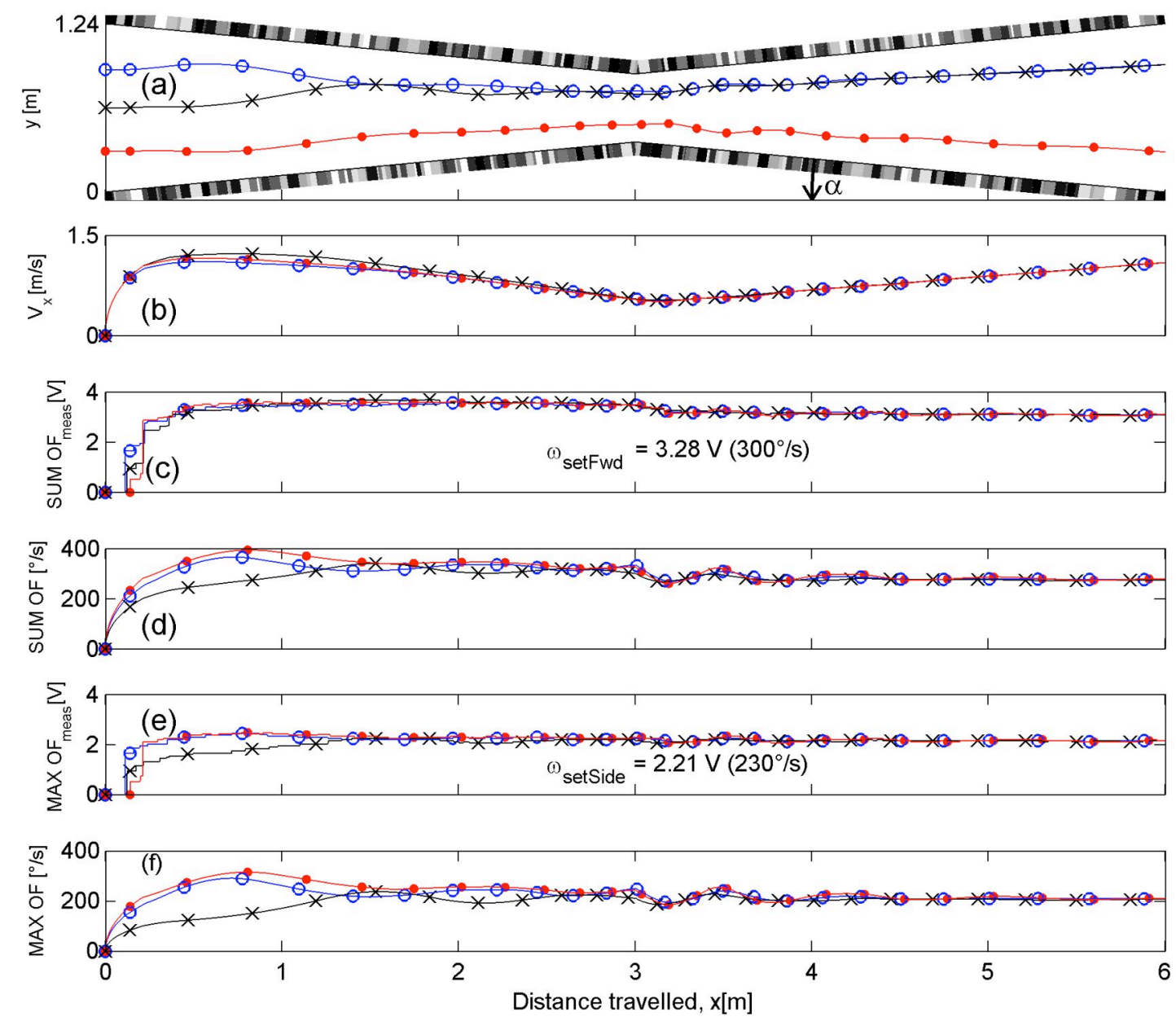

Fig. 12 